\title{
Chance-Constrained Model Predictive Control for Drinking Water Networks
}

\author{
J.M. Grosso*, C. Ocampo-Martínez, V. Puig, B. Joseph \\ Automatic Control Department, Universitat Politècnica de Catalunya (UPC), \\ Institut de Robòtica i Informàtica Industrial (CSIC-UPC), Llorens i Artigas, 4-6, 08028 \\ Barcelona, Spain
}

\begin{abstract}
This paper addresses a chance-constrained model predictive control (CC-MPC) strategy for the management of drinking water networks (DWNs) based on a finite horizon stochastic optimisation problem with joint probabilistic (chance) constraints. In this approach, water demands are considered additive stochastic disturbances with non-stationary uncertainty description, unbounded support and known (or approximated) quasi-concave probabilistic distribution. A deterministic equivalent of the stochastic problem is formulated using Boole's inequality to decompose joint chance constraints into single chance constraints and by considering a uniform allocation of risk to bound these later constraints. The resultant deterministic-equivalent optimisation problem is suitable to be solved with tractable quadratic programming (QP) or second order cone programming (SOCP) algorithms. The reformulation allows to explicitly and easily propagate uncertainty over the prediction horizon, and leads to a cost-efficient management of risk that consists in a dynamic back-off to avoid frequent violation of constraints. Results
\end{abstract}

Email address: jgrosso@iri.upc.edu (J.M. Grosso*) 
of applying the proposed approach to a real case study -the Barcelona DWN (Spain)- have shown that the network performance (in terms of operational costs) and the necessary back-off (to cope with stochastic disturbances) are optimised simultaneously within a single problem, keeping tractability of the solution, even in large-scale networks. The general formulation of the approach and the automatic computation of proper back-off within the MPC framework replace the need of experience-based heuristics or bi-level optimisation schemes that might compromise the trade-off between profits, reliability and computational burden.

Keywords: MPC, drinking water networks, reliability, chance constraints, robustness.

\section{Introduction}

Drinking Water Networks (DWNs) form the link between urban water supply systems and drinking water consumers. These networks are vital for the normal functioning of modern society and maintaining a truly sustainable service is a must in these systems. All water supply undertakings should share a common purpose, stated in [1] as the achievement of the highest level of consumer satisfaction and service quality in line with the prevailing regulatory framework, whilst making best use of available resources. DWNs are large-scale multi-source/multi-sink flow systems that must be reliable and resilient while being subject to constraints and to continuously varying conditions with both deterministic and probabilistic nature. Customers behaviour determines the transport and storage operations within the network. Water use can vary in both the long and the short term, usually presenting time- 
based patterns for different areas. Therefore, a better understanding and forecasting of demands will improve both modelling and control of DWNs.

The growing complexity of these network systems, i.e., dimensionality, information structure constraints, non-linearities, uncertainty, and the higher performance requirements make these kind of problems costly to solve for real-time control applications and their optimal management is a task that has become an increasingly environmental and socio-economic research subject worldwide. Different approaches reported in the literature highlight the importance and development of the topic. As discussed in [2,3], during the last years, optimal operation of water supply systems has been addressed by a wide variety of methods. For example, in [4] a dynamic programming approach is proposed to generate pump schedules in real-time operation of a water supply system. The problem is solved by considering deterministic disturbances and decomposing the system in space and time to apply progressive optimality. In [2] a detailed review of several stochastic dynamic programming techniques applied to water reservoir operations is discussed, highlighting the curse of dimensionality of such techniques and proposing alternative methods to design cyclostationary daily control policies based on reinforcement learning. In [5] a critiquing-expert method that evaluates operating plans and provides feedback to the decision manager is proposed, which includes suggestions for improvement, warnings, and alternatives. In [6] a deterministic linear goal programming method is examined to aid in the identification of optimum operating policies for a multiple-reservoir system, highlighting the importance of the forecast quality to minimise pumping cost, but without robustness consideration. In [7] a combinatorial optimisation 
is proposed for the scheduling of on-off pumps assuming also deterministic and reliable forecast of demands. In [8] a centralised MPC strategy using mixed integer non-linear programming is proposed to regulate water volume in storage tanks and chlorine concentration, both around fixed targets, considering an anticipated consumer demand profile from historical data. Similarly, in [9-11] the economic operation of water networks is performed by using centralised MPC. They also assumed predicted disturbances as certain in the model but included a soft constraint to penalise depletions of water volume below a heuristic safety threshold without forcing any target regulation. An enhancement for these latter methods is proposed in [12], where MPC is combined in a hierarchical way with soft-computing methods and supply chain theory to compute dynamic safety stocks that cope with forecast uncertainty and achieve a self-tuning trade-off between economic optimisation and service reliability of a DWN.

Among the aforementioned approaches, decision policies based on the MPC framework [13], are suitable to face the operation of DWNs given their flexibility to manage constraints and to optimise multi-objective problems as the ones encountered in these complex systems, see [14]. The basic idea of MPC is to exploit a model of the system to predict its future evolution and compute control actions by optimising a desired cost function that takes into account such predictions; if future disturbances can be identified and described, a robust MPC can be developed to explicitly consider their effect on the future evolution of the controlled system.

Decision-making under uncertainty is a central issue in almost all disciplines and application areas. Since uncertainty in DWNs might be large 
and caused by many sources (e.g., exogenous and endogenous demands, noise, equipment degradation, plant model mismatch, other disturbances), it cannot be neglected in optimisation tasks if it is desired to fulfil reliability requirements and quality standards. In industrial practice, uncertainties are usually compensated by over-design of elements or overestimation of operational parameters by introducing safety factors obtained mostly by experience or application-dependent heuristics.

Most of the aforementioned operational MPC strategies for DWNs address uncertainty by solving a deterministic optimisation problem where, following the certainty equivalence principle, stochastic disturbances are replaced by their estimates based upon the information available at each time instant and assuming predictions as certain. This principle guarantees to obtain optimal control actions when using the expectation of disturbances, especially for linear models with small additive uncertainties, Gaussian distributions and quadratic cost functions. The MPC approaches following the aforesaid principle are often denoted certainty equivalent MPC (CE-MPC). Nevertheless, uncertainty in DWNs could be large, which avoids to take for granted that a certainty equivalence is justified for a reliable operation of the constrained system. Hence, the strategy is usually complemented with a (de)tuning of the controller, even though, CE-MPC can lead to poor performance and constraint violations due to the ignored effects of future uncertainty.

There is another widely reported class of control techniques that face uncertainties explicitly in the control law, named the robust model predictive control (RMPC). These strategies use an uncertain process model instead of 
a nominal one. A crucial factor in the design of these RMPC controllers is the characterisation of the uncertainty, which can be divided in two main paradigms: the deterministic worst-case description and the stochastic description. The approach relying on a purely deterministic unknown-butbounded description of the uncertainty has prevailed in the robust control literature, see [15-19], but the main disadvantage of the related strategies is the conservatism of the resultant control policy that negatively affects the utility function of the DWNs operation. Moreover, in real applications the boundedness assumption of disturbances might not hold, hence, constraint violations are unavoidable due to unexpected events, faults, etc.

A more realistic description of uncertainty is the stochastic paradigm, which leads to less conservative control approaches by including explicit models of disturbances in the design of control laws and by transforming hard constraints into probabilistic constraints to cope with inevitable uncertainties. The stochastic approach is a classic one in the field of optimisation (see [20] for a review), but due to the advances in technology, which have improved computation capacity, and due to the flexibility of the MPC framework to incorporate models and constraints within an optimal control problem, a renewed attention has been given to the stochastic programming as a powerful tool for robust control design, leading to the Stochastic $M P C$, which has a particular variant called Chance-Constrained MPC (CC-MPC) [21]. This stochastic control strategy describes robustness in terms of probabilistic (chance) constraints [22], which require that the violation probability of any operational requirement or physical constraint is below a prescribed value, representing the notion of reliability or risk of the DWN. By setting this value 
properly, the operator can trade conservatism against performance. Relevant works that address the CC-MPC approach in water systems can be found in $[23,24]$ and references therein. Other stochastic approaches currently researched are the ones based on multi-stage stochastic programming (MSP) methods, e.g., scenario-based stochastic programming, sampling stochastic dynamic programming, interval stochastic programming, among others [25, 26]. The main limitation of these latter approaches is their narrowed applicability to large-scale stochastic models, which might be a cumbersome task, especially when several disturbances, sources of uncertainty and decision vectors of large dimension are involved. In fact, most of the case studies reported for real-time optimisation of water systems and other applications are small-scale problems, hence, the vast portfolio of developed stochastic techniques has been dedicated for long-term off-line planning of operations or for networks design.

The main contribution of this paper is the introduction of a formal RMPC formulation for the management of DWNs based on chance constraint programming. The paper presents the results of applying an economic CC-MPC to optimise the operation of flow networks, especially those related to the transport and storage of potable water, seeking to achieve a specified customer service level and a reliable DWN. The complexity of the stochastic problem is addressed by using an analytical approximation of the chance constraints to reformulate the problem into a tractable deterministic equivalent by using Boole's inequality, a uniform risk allocation policy and the stochastic characteristics of disturbance forecasts. This systematic approach keeps the convex nature of the multi-objective constrained finite horizon optimisation 
problem and brings other practical benefits, i.e., flexibility, reliability, and tractability of the reformulated CC-MPC controller as a decision-support tool, which are shown in this paper through a real case study: the Barcelona DWN. The approach avoids the set-up of conservative and heuristic thresholds or bi-level optimisation approaches [27] for safety volumes in water storage tanks. In fact, the robustness of the CC-MPC leads to a cost-efficient management of a dynamic back-off (uncertainty-aware time-varying safety volumes) to avoid frequent violation of constraints.

The reminder of this paper is organised as follows. Section 2 describes a control-oriented flow-based model of DWNs and states the CC-MPC problem. Section 3 describes the methodology to obtain a deterministic equivalent of a stochastic MPC with joint chance constraints. Section 4 describes the case study where the effectiveness of the proposed approach is shown and analysed. Section 5 highlights the concluding remarks that can be drawn from the results presented in this paper as well as some future research directions.

\section{Notation}

Throughout this paper, let $\mathbb{R}, \mathbb{R}^{n}, \mathbb{R}^{m \times n}$, denote the field of real numbers, the set of column real vectors of length $n$ and the set of $m$ by $n$ real matrices, respectively, whereas $\mathbb{N} \triangleq\{0,1,2, \ldots\}$ is the set of natural numbers including zero, $\mathbb{N}_{+} \triangleq \mathbb{N} \backslash\{0\}$, and $\mathbb{N}_{j}^{k}$ is the set of consecutive non-negative integers $\{j, \ldots, k\}$. Scalars are denoted with lower case letters (e.g., $a, b, \alpha, \beta, \ldots)$, vectors are denoted with bold lower case letters (e.g., a, b, ..), matrices are denoted with bold upper case letters (e.g., A, B,...), and general sets are denoted with upper case calligraphic letters (e.g., $\mathcal{A}, \mathcal{B}, \ldots$ ). The cardinal of 
a set $\mathcal{A}$ is denoted with $|\mathcal{A}|$. If not otherwise noted, all vectors are column vectors. Moreover, $\mathbf{0}$ denotes a zero column vector and $\mathbf{I}$ the identity matrix, both of appropriate dimensions, while $\mathbf{v}_{\rightarrow} \triangleq\left[\mathbf{v}_{k \mid k}, \ldots, \mathbf{v}_{k+N \mid k}\right] \in \mathbb{R}^{n_{v} \times N}$ denotes an ordered collection of $N$ predicted vectors $\mathbf{v}_{k+i \mid k} \in \mathbb{R}^{n_{v}}$. By superscript ${ }^{\mathrm{T}}$ transposition is denoted, and the operators $<, \leq,=,>, \geq$ denote element-wise relations of vectors.

\section{Problem Formulation}

\subsection{DWN flow-based control-oriented model}

This paper considers a general DWN being represented by a directed graph $G(\mathcal{V}, \mathcal{E})$, see [28], where a set of elements, i.e., $n_{s}$ sources, $n_{x}$ storage elements, $n_{q}$ intersection nodes, and $n_{d}$ sinks, are represented by $v \in \mathcal{V}$ vertices, which are connected by $a \in \mathcal{E}$ directed links. Due to the network function, water is transported along the links by $n_{u}$ flow actuators (i.e., pipes and valves), passing through reservoirs or tanks, from specific origin locations to specific destination locations. The network is subject to several capacity and operational constraints, and to measured stochastic flows to sinks driven by customers water demand.

Stating the volume in storage elements as the state variable $\mathbf{x}_{k} \in \mathbb{R}^{n_{x}}$, the flow through the actuators as the manipulated inputs $\mathbf{u}_{k} \in \mathbb{R}^{n_{u}}$, and the demanded flow as additive measured disturbances $\mathbf{d}_{k} \in \mathbb{R}^{n_{d}}$, then the controloriented model of the DWN $G(\mathcal{V}, \mathcal{E})$ may be abstracted and described by the following set of linear (or linearised) discrete difference-algebraic equations 
(DAE) for all time instant $k \in \mathbb{N}$ :

$$
\begin{aligned}
\mathbf{x}_{k+1} & =\mathbf{A} \mathbf{x}_{k}+\mathbf{B} \mathbf{u}_{k}+\mathbf{B}_{d} \mathbf{d}_{k} \\
\mathbf{0} & =\mathbf{E}_{u} \mathbf{u}_{k}+\mathbf{E}_{d} \mathbf{d}_{k}
\end{aligned}
$$

where the difference equations in (1a) describe the dynamics of storage tanks, and the algebraic equations in (1b) describe the static relations (i.e., mass balance at junction nodes) in the network. Moreover, $\mathbf{A}, \mathbf{B}, \mathbf{B}_{d}, \mathbf{E}_{u}, \mathbf{E}_{d}$, are time-invariant matrices of suitable dimensions dictated by the network topology.

Assumption 1. The states in $\mathbf{x}_{k}$ and the demands in $\mathbf{d}_{k}$ are observable at time instant $k$, and the pair $(\mathbf{A}, \mathbf{B})$ is stabilisable.

Assumption 2. The realisation of disturbances at the current time instant $k$ may be decomposed as

$$
\mathbf{d}_{k}=\overline{\mathbf{d}}_{k}+\tilde{\mathbf{d}}_{k}
$$

where $\overline{\mathbf{d}}_{k}$ is the vector of expected disturbances, and $\tilde{\mathbf{d}}_{k}$ is the vector of probabilistic independent forecasting errors with non-stationary uncertainty and a known (or approximated) quasi-concave probability distribution $\mathcal{D}$, e.g., the normal, the logistic, the exponential distribution, among others. Therefore, the stochastic nature of each $i^{\text {th }}$ row of $\mathbf{d}_{k}$ is described by $\mathbf{d}_{(i), k} \sim$ $\mathcal{D}_{(i)}\left(\overline{\mathbf{d}}_{(i), k}, \Sigma_{\tilde{\mathbf{d}}_{(i)}, k}\right)$, where $\overline{\mathbf{d}}_{(i), k}$ denotes its mean, and $\Sigma_{\tilde{\mathbf{d}}_{(i)}, k}$ its variance. Given the past $k$ measured realisations of $\mathbf{d}_{k}$, its stochastic description can be predicted for $N$ time instants ahead, using, e.g., time-series models.

The system is subject to hard state and input constraints given by convex 
and closed polytopic sets defined as

$$
\begin{aligned}
& \mathcal{X} \triangleq\left\{\mathbf{x}_{k} \in \mathbb{R}^{n_{x}} \mid \mathbf{G}_{k} \leq \mathbf{g}\right\} \subset \mathbb{R}^{n_{x}} \quad \forall k \\
& \mathcal{U} \triangleq\left\{\mathbf{u}_{k} \in \mathbb{R}^{n_{u}} \mid \mathbf{F} \mathbf{u}_{k} \leq \mathbf{f}\right\} \subset \mathbb{R}^{n_{u}} \quad \forall k
\end{aligned}
$$

where $\mathbf{G} \in \mathbb{R}^{c_{x} \times n_{x}}, \mathbf{g} \in \mathbb{R}^{c_{x}}, \mathbf{F} \in \mathbb{R}^{c_{u} \times n_{u}}, \mathbf{f} \in \mathbb{R}^{c_{u}}$, being $c_{x}$ and $c_{u}$ the number of state and input constraints, respectively.

Notice in (1) that some control variables are directly related with measured disturbances. Hence, it is clear that $\mathbf{u}$ does not take values in $\mathbb{R}^{n_{u}}$ but in a linear variety given by the algebraic equation (1b). As shown in [29], this latter observation can be exploited to develop an affine parametrisation of control variables in terms of a minimum set of disturbances, mapping control problems to a space with a smaller decision vector and with less computational burden due to the elimination of the equality constraints. Thus, the model is rewritten as

$$
\mathbf{x}_{k+1}=\mathbf{A} \mathbf{x}_{k}+\tilde{\mathbf{B}} \tilde{\mathbf{u}}_{k}+\tilde{\mathbf{B}}_{d} \mathbf{d}_{k}
$$

and constraints in (3b) are replaced with a time-varying restricted input constraint set defined as

$$
\tilde{\mathcal{U}}_{k} \triangleq\left\{\tilde{\mathbf{u}}_{k} \in \mathbb{R}^{n_{\tilde{u}}} \mid \mathbf{F} \tilde{\mathbf{P}} \tilde{\mathbf{M}}_{1} \tilde{\mathbf{u}}_{k} \leq \mathbf{f}-\mathbf{F} \tilde{\mathbf{P}} \tilde{\mathbf{M}}_{2} \mathbf{d}_{k}\right\} \subset \mathbb{R}^{n_{\tilde{u}}} \quad \forall k
$$

(see Appendix A).

Given the stochastic nature of disturbances, state constraints are relaxed with a predefined probability to manage the DWN reliability as follows:

$$
\mathbb{P}\left[\mathbf{x}_{k} \in \mathcal{X}\right] \geq 1-\delta_{x}
$$


where $\delta_{x} \in(0,1)$ denotes the risk probability of constraint violation for the states. This probabilistic general constraint is the so called chance constraint. There are two forms of chance constraints according to the definitions below.

Definition 1 (Joint chance constraint). A (linear) joint chance constraint is of the form

$$
\mathbb{P}\left[\mathbf{G}_{(j)} \mathbf{x}_{k} \leq \mathbf{g}_{(j)}, \forall j \in \mathbb{N}_{1}^{c_{x}}\right] \geq 1-\delta_{x} \quad \forall k
$$

where $\mathbf{G}_{(j)}$ and $\mathbf{g}_{(j)}$ denote the $j^{\text {th }}$ row of $\mathbf{G}$ and $\mathbf{g}$, respectively. This requires that all rows $j$ have to be jointly fulfilled with the probability $1-\delta_{x}$.

Definition 2 (Individual chance constraint). A (linear) individual chance constraint is of the form

$$
\mathbb{P}\left[\mathbf{G}_{(j)} \mathbf{x}_{k} \leq \mathbf{g}_{(j)}\right] \geq 1-\delta_{x, j}, \quad \forall j \in \mathbb{N}_{1}^{c_{x}} \quad \forall k
$$

which requires that each $j^{\text {th }}$ row of the inequality has to be fulfilled individually with the respective probability $1-\delta_{x, j}$, where $\delta_{x, j} \in(0,1)$.

Both forms of constraints are useful to measure risks, hence, their selection depends on the application. This paper is concerned with the use of joint chance constraints since they can express better the management of the overall reliability in a DWN. All chance-constrained models require prior knowledge of the acceptable risk associated with the constraints. A lower risk acceptability implies a harder constraint.

\subsection{Chance-Constrained Model Predictive Control}

This paper addresses the problem of finding, over a fixed prediction horizon $H_{p} \in \mathbb{N}_{+}$, a sequence of control actions $\underline{\tilde{\mathbf{u}}}_{k}^{*}\left(\mathbf{x}_{k}, \mathbf{d}_{k}\right) \triangleq\left[\tilde{\mathbf{u}}_{k \mid k}^{*}, \ldots, \tilde{\mathbf{u}}_{k+H_{p}-1 \mid k}^{*}\right]$ 
that allows to redistribute resources within a DWN, from the times and places where they are available to the ones where they are needed. This must be done in such a way that a set $\mathcal{O}$ of (managers dependant) objectives is optimised subject to input polytopic constraints and state joint chance constraints that cope with the stochastic component of demands. From the model in $(1)$, let $\underline{\mathbf{d}} \triangleq\left(\mathbf{d}_{k}, \mathbf{d}_{k+1 \mid k}, \ldots, \mathbf{d}_{k+H_{p}-1 \mid k}\right)$ be the sequence of disturbances over a fixed time prediction horizon $H_{p}$. The first element of the sequence is measured, while the rest of the elements, i.e., $\left\{\mathbf{d}_{k+i \mid k}\right\}_{i=1}^{H_{p}-1}$, denote estimates of future disturbances computed by an exogenous system and available at each time instant $k$. Hence, the CC-MPC controller design is based on the solution of the following multi-objective finite horizon optimisation problem (FHOP).

\section{Problem 1 (CC-FHOP for DWNs).}

$$
\min _{\tilde{\tilde{\mathbf{u}}}_{k}^{*}} \mathbb{E}\left[\sum_{m=1}^{|\mathcal{O}|} \sum_{i=0}^{H_{p}-1} \lambda_{m} J_{m}\left(\mathbf{x}_{k+i+1 \mid k}, \tilde{\mathbf{u}}_{k+i \mid k}, \mathbf{d}_{k+i \mid k}\right)\right],
$$

subject to:

$$
\begin{aligned}
& \mathbf{x}_{k+i+1 \mid k}=\mathbf{A} \mathbf{x}_{k+i \mid k}+\tilde{\mathbf{B}} \tilde{\mathbf{u}}_{k+i \mid k}+\tilde{\mathbf{B}}_{d} \mathbf{d}_{k+i \mid k}, \\
& \tilde{\mathbf{u}}_{k+i \mid k} \in \tilde{\mathcal{U}}_{k+i \mid k}, \\
& \mathbb{P}\left[\bigcap_{i=0}^{H_{p}-1} \mathbf{x}_{k+i+1 \mid k} \in \mathcal{X}\right] \geq 1-\delta_{x}, \\
& \left(\mathbf{x}_{k \mid k}, \mathbf{d}_{k \mid k}\right)=\left(\mathbf{x}_{k}, \mathbf{d}_{k}\right),
\end{aligned}
$$

where $\mathbb{E}[\cdot]$ and $\mathbb{P}[\cdot]$ are the expectation and probability operators, respectively, and $\delta_{x}$ denotes the upper bound of the joint risk for constraints violation. 
The expectation of the cost function is over the states and disturbances, and the optimisation is over the sequence of control inputs. Each $J_{m}$ objective considered in the above FHOP is prioritised within the overall cost function through $m$ positive scalar weights $\lambda_{m}$.

Assuming that Problem 1 is feasible, i.e., $\underline{\mathbf{u}}_{k}^{*} \neq \emptyset$, and taking into account the MPC law [13] and (A.10), then the controller applies the input given by

$$
\mathbf{u}_{\mathrm{MPC}, k} \triangleq \tilde{\mathbf{P}} \tilde{\mathbf{M}}_{1} \tilde{\mathbf{u}}_{k \mid k}^{*}+\tilde{\mathbf{P}} \tilde{\mathbf{M}}_{2} \mathbf{d}_{k} .
$$

In general, (9) could be non-convex depending on the probability distributions involved in the chance constraints. The main challenge in solving this stochastic problem lies in computing the multivariate integration of the density function of the uncertain variables. As reviewed in [30], this can be done by two general approaches: (i) analytical methods or (ii) sampling methods. In this paper, the former approach is considered by addressing analytically the stochastic process of disturbances (using time-series modelling) and assuming an uncertainty description given by a multivariate Gaussian distribution to exploit its properties and reformulate the chance constraints into a set of deterministic equivalents.

\section{Deterministic Equivalent CC-MPC}

\subsection{Tractable Safe Approximation of Joint Chance Constraints}

\subsubsection{Convexity Analysis}

Constraint (9d) of Problem 1 requires that the probability of violating any of multiple inequality state constraints is below a user-defined bound. Thus, such probabilistic constraint is a joint chance constraint. Consider that this 
constraint can be generalised in a vector function $\mathbf{h}(\mathbf{v}, \boldsymbol{\zeta}): \mathbb{R}^{n_{v} \times n_{\zeta}} \mapsto \mathbb{R}^{n_{c}}$, where a random vector $\boldsymbol{\zeta} \in \mathbb{R}^{n_{\zeta}}$ is separable of a decision vector $\mathbf{v} \in \mathbb{R}^{v}$ taking into account that the uncertainty appears in (1) in an additive way. Hence, the joint chance constraint can be expressed in a general form as $\mathbb{P}[\mathbf{h}(\mathbf{v}, \boldsymbol{\zeta}) \leq \mathbf{0}] \geq 1-\delta_{\zeta}$, where $\delta_{\zeta} \in(0,1)$ is the risk bound. As demonstrated in [31], if $\mathbf{h}(\cdot, \cdot)$ is jointly convex in $(\mathbf{v}, \boldsymbol{\zeta})$ and $\Phi \triangleq \mathbb{P}[\cdot]$ is quasi-concave, then the feasible set $\mathcal{B}\left(\delta_{\zeta}\right) \triangleq\left\{\mathbf{v} \mid \mathbb{P}[\mathbf{h}(\mathbf{v}, \boldsymbol{\zeta}) \leq \mathbf{0}] \geq 1-\delta_{\zeta}\right\}$ is convex $\forall \delta_{\zeta} \in(0,1)$.

Recalling that the original deterministic constraint (3a) forms a convex and closed set, and under the assumption that disturbance uncertainty lies in a multivariate distribution whose probabilistic density function $\Phi$ is logconcave, then it can be concluded that Problem 1 is a smooth convex program.

\subsubsection{Decomposition of Joint Chance Constraints}

Although the joint chance constraint (9d) is convex, the evaluation of its left-hand side is still necessary. In general, joint chance constraints lack analytical expressions; the available methods to integrate the involved multivariate probability distribution over an arbitrary region, e.g., samplingbased methods or numeric integration, may be intractable for large-scale systems. Nevertheless, there are some approaches that solve the issue by bounding or approximating the exact numeric solution, see [32] and references therein. Here, a uniform distribution of the joint risk is distributed among a set of individual chance constraints that can be transformed, under the assumption of log-concavity of their univariate distributions, into equivalent deterministic constraints that can be evaluated analytically.

The decomposition approach is as follows. Consider again the general 
joint chance constraint, i.e., $\mathbb{P}[(\mathbf{v}, \boldsymbol{\zeta}) \leq \mathbf{0}] \geq 1-\delta_{\zeta}$, and define $\mathbf{h}(\mathbf{v}, \boldsymbol{\zeta}) \triangleq \boldsymbol{\zeta}-\mathbf{H v}$, with $\boldsymbol{\zeta} \in \mathbb{R}^{n_{\zeta}}, \mathbf{v} \in \mathbb{R}^{n_{v}}$, and $\mathbf{H} \in \mathbb{R}^{n_{\zeta} \times n_{v}}$. Hence, the additive stochastic element is separable and the following chance constraint is obtained:

$$
\mathbb{P}[\boldsymbol{\zeta} \leq \mathbf{H v}] \geq 1-\delta_{\zeta} .
$$

To simplify the notation, define $\mathbf{w} \triangleq \mathbf{H v} \in \mathbb{R}^{n_{c}}$, then, for any duple $\langle\boldsymbol{\zeta}, \mathbf{w}\rangle$, it follows that

$$
\Phi_{\zeta}(\mathbf{w})=\mathbb{P}\left[\left\{\zeta_{1} \leq w_{1}, \ldots, \zeta_{n_{c}} \leq w_{n_{c}}\right\}\right] .
$$

Defining the events $C_{i} \triangleq\left\{\zeta_{i} \leq w_{i}\right\}, \forall i \in \mathbb{N}_{1}^{n_{c}}$, it follows:

$$
\Phi_{\zeta}(\mathbf{w})=\mathbb{P}\left[C_{i} \cap \ldots \cap C_{n_{c}}\right] .
$$

Denoting the complements of the events $C_{i}$ by

$$
C_{i}^{c} \triangleq\left\{\zeta_{i}>w_{i}\right\}
$$

it is known from probability theory that

$$
C_{1} \cap \ldots \cap C_{n_{c}}=\left(C_{1}^{c} \cup \ldots \cup C_{n_{c}}^{c}\right)^{c},
$$

and consequently

$$
\begin{aligned}
\Phi_{\zeta}(\mathbf{w}) & =\mathbb{P}\left[C_{1} \cap \ldots \cap C_{n_{c}}\right] \\
& =\mathbb{P}\left[\left(C_{1}^{c} \cup \ldots \cup C_{n_{c}}^{c}\right)^{c}\right] \\
& =1-\mathbb{P}\left[\left(C_{1}^{c} \cup \ldots \cup C_{n_{c}}^{c}\right)\right] \geq 1-\delta_{\zeta} .
\end{aligned}
$$

At this point, taking advantage of the union bound, the Boole's inequality allows to bound the result in (16c), stating that for a countable set of events 
$E_{1}, \ldots, E_{n_{c}}$, the probability that at least one event happens is not higher than the sum of the individual probabilities [33], i.e.,

$$
\mathbb{P}\left[\bigcup_{i=1}^{n_{c}} E_{i}\right] \leq \sum_{i=1}^{n_{c}} \mathbb{P}\left[E_{i}\right] .
$$

Defining $E_{i} \triangleq C_{i}^{c}, \forall i \in \mathbb{N}_{1}^{n_{c}}$, and applying (17) to the inequality in (16c), it follows that

$$
\sum_{i=1}^{n_{c}} \mathbb{P}\left[C_{i}^{c}\right] \leq \delta_{\zeta} \Leftrightarrow \sum_{i=1}^{n_{c}}\left(1-\mathbb{P}\left[C_{i}\right]\right) \leq \delta_{\zeta} .
$$

At this point, a set of constraints arises from previous results as sufficient conditions to enforce the joint chance constraint (11), by allocating the joint risk $\delta_{\zeta}$ in $n_{c}$ separate risks $\delta_{\zeta, i}, i \in \mathbb{N}_{1}^{n_{c}}$. These constraints are stated as follows:

$$
\begin{aligned}
& \mathbb{P}\left[C_{i}\right] \geq 1-\delta_{\zeta, i}, \quad \forall i \in \mathbb{N}_{1}^{n_{c}}, \\
& \sum_{i=1}^{n_{c}} \delta_{\zeta, i} \leq \delta_{\zeta}, \\
& 0 \leq \delta_{\zeta, i} \leq 1,
\end{aligned}
$$

where (19) forms the set of $n_{c}$ resultant individual chance constraints, which bounds the probability that each inequality of the receding horizon problem may fail; and (20) and (21) are conditions imposed to bound the new single risks in such a way that the joint risk bound is not violated. Any solution that satisfies the above constraints guarantees to satisfy (11).

Remark 1. The bounds for the risk probability levels of joint or individual chance constraints mentioned in this paper apply for the case when the uncertainty is in the separable vector $\boldsymbol{\zeta}$. If the random component is the 
matrix $\mathbf{H}$, then the bounds for the risk probability, to keep a convex problem, may change, e.g., for Gaussian distribution $\delta \in(0,0.5]$.

\subsubsection{Risk Allocation Policy}

The decomposition of the joint chance constraint into single constraints leads to the incorporation of $n_{c}$ new variables (the individual risks) in the problem, thus, the calculation of their value has to be addressed. In the literature, two methods have been proposed to solve this issue: (i) fixed-risk method [34], and (ii) optimal risk allocation method [30, 35]. The former method assigns a pre-defined constant risk for each univariate constraint in (19), typically following a uniform allocation strategy. The latter method assumes the single risks as new decision variables to be optimised, what improves the performance of the overall system but at the cost of more computational burden due to the greater complexity and dimensionality of the optimisation task. Therefore, as DWNs are often large-scale systems, the uniform risk allocation policy is adopted here to avoid overloading of the associated optimisation problem.

Consider the joint chance constraint (11) with a predefined allowed probability of violation given by the accepted maximal risk $\delta_{\zeta}$. After the decomposition of such constraint into $n_{c}$ individual chance constraints, the uniform risk allocation policy assigns a fixed and equal value of risk to each constraint as follows:

$$
\delta_{\zeta, i}=\frac{\delta_{\zeta}}{n_{c}}, \quad \forall i \in \mathbb{N}_{1}^{n_{c}} .
$$

In the case of the MPC strategy, the joint chance constraints may lead to a large number of individual chance constraints because $n_{c}$ depends not only on the number of constraints that are set up, but also on the prediction 
horizon over which those constraints have to be evolved using the prediction models. Then, the joint chance constraint (9d) in Problem 1 leads to $n_{c} \times H_{p}$ individual chance constraints. This fact reinforces the use of the fixed risk distribution policy for DWNs control problems, in order to avoid the addition of a large number of new decision variables to be optimised.

Remark 2. The constraint bound $1-\delta_{\zeta}$ is usually assigned a priori by the decision makers and represents the desired probability of maintaining the feasibility of the optimisation problem. In general, high levels of risk $\delta_{\zeta}$ associated with satisfying the constraints lead to obtain higher cost function values.

\subsubsection{Deterministic Equivalent Constraints}

A chance constraint is not suitable for algebraic solution, thus, after decomposing the joint constraints into a set of individual constraints, the deterministic equivalent of each separate constraint should be used. Reminding that this paper deals with additive uncertainty, such deterministic equivalents are obtained following [36] and described below.

Consider (19) and the set of $n_{c}$ individual chance constraints expressed as

$$
\mathbb{P}\left[\boldsymbol{\zeta}_{(i)} \leq \mathbf{H}_{(i)} \mathbf{v}\right] \geq 1-\delta_{\zeta, i}, \quad \forall i \in \mathbb{N}_{1}^{n_{c}}
$$

where $\boldsymbol{\zeta}_{(i)}$ is the $i^{\text {th }}$ row of the separable random vector $\boldsymbol{\zeta}$ with Gaussian uncertainty $\boldsymbol{\zeta}_{(i)} \sim \mathcal{N}\left(\overline{\boldsymbol{\zeta}}_{(i)}, \Sigma_{\boldsymbol{\zeta}_{(i)}}\right)$, and $\mathbf{H}_{(i)}$ is the $i^{\text {th }}$ row of the matrix $\mathbf{H} \in \mathbb{R}^{n_{c} \times n_{v}}$. Using the standardised variate of the random vector, i.e.,

$$
Z_{\zeta_{i}}=\frac{\boldsymbol{\zeta}_{(i)}-\overline{\boldsymbol{\zeta}}_{(i)}}{\Sigma_{\zeta_{i}}^{1 / 2}}
$$


then (23) can be expressed as

$$
\mathbb{P}\left[Z_{\zeta_{i}} \leq \frac{\mathbf{H}_{(i)} \mathbf{v}-\overline{\boldsymbol{\zeta}}_{(i)}}{\Sigma_{\zeta_{i}}^{1 / 2}}\right] \geq 1-\delta_{\zeta, i} \Longleftrightarrow \Phi\left(\frac{\mathbf{H}_{(i)} \mathbf{v}-\overline{\boldsymbol{\zeta}}_{(i)}}{\Sigma_{\zeta_{i}}^{1 / 2}}\right) \geq 1-\delta_{\zeta, i}
$$

where $\overline{\boldsymbol{\zeta}}_{(i)}$ and $\Sigma_{\zeta_{i}}$ are the mean and the variance of the $i^{\text {th }}$ element of $\boldsymbol{\zeta}$. Moreover, $\Phi$ is the standard Gaussian cumulative distribution function and its inverse $\Phi^{-1}$ (the quantile function) allows to finally derive the deterministic equivalent of (23) in the form of

$$
\mathbf{H}_{(i)} \mathbf{v} \geq \overline{\boldsymbol{\zeta}}_{(i)}+\Phi^{-1}\left(1-\delta_{\zeta, i}\right) \Sigma_{\zeta_{i}}^{1 / 2}, \quad \forall i \in \mathbb{N}_{1}^{n_{c}}
$$

In this way, the reformulated predictive controller solves the deterministic equivalent FHOP stated below.

Problem 2 (Deterministic Equivalent CC-FHOP for DWNs).

$$
\min _{\tilde{\tilde{\mathbf{u}}}_{k}^{*}}: \mathbb{E}\left[\sum_{m=1}^{|\mathcal{O}|} \sum_{i=0}^{H_{p}-1} \lambda_{m} J_{m}\left(\mathbf{x}_{k+i+1 \mid k}, \tilde{\mathbf{u}}_{k+i \mid k}, \mathbf{d}_{k+i \mid k}\right)\right],
$$

subject to:

$$
\begin{aligned}
& \overline{\mathbf{x}}_{k+i+1 \mid k}=\mathbf{A} \overline{\mathbf{x}}_{k+i \mid k}+\tilde{\mathbf{B}} \tilde{\mathbf{u}}_{k+i \mid k}+\tilde{\mathbf{B}}_{d} \overline{\mathbf{d}}_{k+i \mid k}, \\
& \tilde{\mathbf{u}}_{k+i \mid k} \in \tilde{\mathcal{U}}_{k+i \mid k}, \\
& \mathbf{G}_{(j)} \overline{\mathbf{x}}_{k+i+1 \mid k} \leq \mathbf{g}_{(j)}-\Phi^{-1}\left(1-\frac{\delta_{x}}{c_{x} H_{p}}\right) \Sigma_{\mathbf{G}_{(j)} \mathbf{x}, k+i+1 \mid k}^{1 / 2} \quad \forall j \in \mathbb{N}_{1}^{c_{x}}, \\
& \left(\overline{\mathbf{x}}_{k \mid k}, \overline{\mathbf{d}}_{k \mid k}\right)=\left(\mathbf{x}_{k}, \mathbf{d}_{k}\right) .
\end{aligned}
$$

Remark 3. It turns out that most (not all) probability distribution functions used in different applications, e.g., uniform, Gaussian, logistic, Chi-squared, 
Gamma, Beta, log-normal, Weibull, Dirichlet, Wishart, among other distributions, share the property of being log-concave. Then, their corresponding quantile function can be computed off-line for a given risk acceptability level and used within the MPC convex optimisation in a similar way than the Gaussian quantile used in this paper.

\subsubsection{Conservatism}

The approach presented above to derive deterministic equivalents of joint chance constraints, gives a conservative approximation of the original stochastic problem. The impact of the conservatism on the quality of the solution is discussed below.

Consider again that the satisfaction of each individual constraint is an event $C_{i}, \forall i \in \mathbb{N}_{1}^{n_{c}}$. A joint chance constraint requires that the conjunction of all the individual constraints is satisfied with a desired probability level $1-\delta_{\zeta}$, i.e.

$$
\mathbb{P}\left[\bigcap_{i=1}^{n_{c}} C_{i}\right] \geq 1-\delta_{\zeta} .
$$

Under the assumption that each individual constraint is probabilistically independent, the probability of the joint constraint, considering the uniform risk allocation policy in (22), is given by

$$
\begin{aligned}
\mathbb{P}\left[\bigcap_{i=1}^{n_{c}} C_{i}\right] & =\prod_{i=1}^{n_{c}} \mathbb{P}\left[C_{i}\right] \\
& =\prod_{i=1}^{n_{c}}\left(1-\delta_{\zeta, i}\right) \\
& =\left(1-\frac{\delta_{\zeta}}{n_{c}}\right)^{n_{c}}
\end{aligned}
$$


Taking into account that using Boole's inequality to upper bound the joint constraint leads to (19), (20) and (21), it follows that

$$
\left(1-\frac{\delta_{\zeta}}{n_{c}}\right)^{n_{c}} \geq\left(1-\delta_{\zeta}\right) \Leftrightarrow \delta_{\zeta} \geq 1-\left(1-\frac{\delta_{\zeta}}{n_{c}}\right)^{n_{c}} .
$$

In this way, the approximated conservatism $\tilde{\Delta}$ introduced by the CC-MPC approach with individual constraints presented in this paper is given by

$$
\tilde{\Delta}=\delta-\left(1-\left(1-\frac{\delta_{\zeta}}{n_{c}}\right)^{n_{c}}\right) .
$$

Remark 4. The level of conservatism without assumptions on the independence of events, can be derived by using the inclusion-exclusion principle for the union of finite events, $E_{i}, \forall i \in \mathbb{N}_{1}^{n_{c}}$, which asserts the following equality:

$$
\begin{aligned}
\mathbb{P}\left[\bigcup_{i=1}^{n_{c}} E_{i}\right]= & \sum_{i=1}^{n_{c}} \mathbb{P}\left[E_{i}\right] \\
& -\sum_{1 \leq i<j \leq n_{c}} \mathbb{P}\left[E_{i} \cap E_{j}\right] \\
& +\sum_{1 \leq i<j<k \leq n_{c}} \mathbb{P}\left[E_{i} \cap E_{j} \cap E_{k}\right] \\
& -\cdots+(-1)^{n_{c}-1} \mathbb{P}\left[\bigcap_{i=1}^{n_{c}} E_{i}\right] .
\end{aligned}
$$

Defining $E_{i} \triangleq C_{i}^{c}$, and subtracting (32) from (17), it follows that the conservatism is given by

$$
\begin{aligned}
\Delta= & \sum_{1 \leq i<j \leq n_{c}} \mathbb{P}\left[C_{i}^{c} \cap C_{j}^{c}\right] \\
& -\sum_{1 \leq i<j<k \leq n_{c}} \mathbb{P}\left[C_{i}^{c} \cap C_{j}^{c} \cap C_{k}^{c}\right]+\cdots-(-1)^{n_{c}-1} \mathbb{P}\left[\bigcap_{i=1}^{n_{c}} C_{i}^{c}\right] .
\end{aligned}
$$


To evaluate the resulting expression requires the knowledge of the conditional probability of the events, which might be even impractical to obtain. Hence, (31) is used as an approximated indicator.

\section{Case Study: The Barcelona Drinking Water Network}

\subsection{General Description}

The approach presented in this paper is assessed with a case study of a real network, specifically the Barcelona DWN. This network is currently managed by $\mathrm{AGBAR}^{1}$ and it supplies drinking water to the Metropolitan Area of Barcelona (Catalunya, Spain). In general, the water network operates as a fully interconnected system driven by endogenous and exogenous flow demands; different hydraulic elements are used to collect, store, distribute and serve drinking water to the associated population.

The operational goals in the management of the Barcelona DWN are of three kinds: economic, safety, smoothness, and are respectively stated as follows:

1. To provide a reliable water supply in the most economic way, minimising water production and transport costs.

2. To guarantee the availability of enough water in each reservoir to satisfy its underlying demand, keeping a safety stock to face uncertainties and avoid stock-outs.

3. To operate the DWN under smooth control actions.

\footnotetext{
${ }^{1}$ Aguas de Barcelona, S.A. Company that manages the drinking water transport and distribution in Barcelona (Spain).
} 
According to Section 2.1, this system can be generally described in statespace form by (4). The states and control inputs are subject to the following hard constraints:

$$
\begin{aligned}
& \mathbf{x}_{\min } \leq \mathbf{x}_{k} \leq \mathbf{x}_{\max }, \quad \forall k, \\
& \mathbf{u}_{\min } \leq \tilde{\mathbf{P}} \tilde{\mathbf{M}}_{1} \tilde{\mathbf{u}}_{k}+\tilde{\mathbf{P}} \tilde{\mathbf{M}}_{2} \mathbf{d}_{k} \leq \mathbf{u}_{\max }, \quad \forall k,
\end{aligned}
$$

where $\mathbf{x}_{\text {min }} \in \mathbb{R}^{n_{x}}$ and $\mathbf{x}_{\max } \in \mathbb{R}^{n_{x}}$ denote the vectors of minimum and maximum volume capacities in tanks, respectively, given in $\mathrm{m}^{3}$; while $\mathbf{u}_{\min } \in \mathbb{R}^{n_{u}}$ and $\mathbf{u}_{\max } \in \mathbb{R}^{n_{u}}$ denote the vectors of minimum and maximum flow capacities through the system actuators, respectively, given in $\mathrm{m}^{3} / \mathrm{s}$. Moreover, for service reliability, the states are also subject to the following safety constraint:

$$
\mathbf{x}_{k} \geq \mathbf{d}_{\text {net }, k}, \quad \forall k
$$

where $\mathbf{d}_{\text {net }, k} \triangleq\left|\mathbf{B}_{\text {out }}\left(\tilde{\mathbf{P}} \tilde{\mathbf{M}}_{1} \tilde{\mathbf{u}}_{k-1}+\tilde{\mathbf{P}} \tilde{\mathbf{M}}_{2} \mathbf{d}_{k-1}\right)+\mathbf{B}_{d} \mathbf{d}_{k}\right| \in \mathbb{R}^{n_{x}}$ is a vector of net demands in $\mathrm{m}^{3}$, above which is desired to keep the reservoirs to avoid stock-outs. The $\mathbf{B}_{\text {out }}\left(\tilde{\mathbf{P}} \tilde{\mathbf{M}}_{1} \tilde{\mathbf{u}}_{k-1}+\tilde{\mathbf{P}} \tilde{\mathbf{M}}_{2} \mathbf{d}_{k-1}\right)$ component represents the immediate previous endogenous demands, i.e., the outflow of the tanks caused by water requirements from neighbouring tanks or nodes, and the $\mathbf{B}_{d} \mathbf{d}_{k}$ component denotes the exogenous (customer) demands of tanks for a given time instant $k$.

The Barcelona DWN flow-based model contains a total amount of 63 tanks and 114 manipulated actuators. Moreover, the network has 88 demand sectors and 17 pipe intersection nodes. For further details about the DWN modelling and specific insights related to this case study, the reader is referred to [37]. 


\section{2. $C C-M P C$ Setup}

Given the stochastic nature of water demands, the DWN prediction model involves exogenous additive uncertainties, hence, the compliance of constraints for a given control input cannot be ensured. This means that, even if the predictive controller finds a feasible solution to achieve the operational goals, there is a certain probability that real outputs may violate constraints. Therefore, it is appropriate to replace the original constraints that involve stochastic elements, i.e., (34a) and (35), by probabilistic statements in the form of chance constraints. In this section, the DWN is considered as a probabilistic constrained system subject to polytopic constraints on the controls and linear joint chance constraints on the states. The source of uncertainty in the system is assumed to be the forecasting error of the measured demands. Then, the stochastic control Problem 1 for the case study is stated as follows for $i \in \mathbb{N}_{0}^{H_{p}-1}$ :

$$
J^{*} \triangleq \min _{\tilde{\tilde{\mathbf{u}}}_{k}^{*}} \mathbb{E}\left[\lambda_{1} \sum_{i=0}^{H_{p}-1} J_{E, k+i \mid k}+\lambda_{2} \sum_{i=0}^{H_{p}-1} J_{\Delta U, k+i \mid k}\right],
$$


subject to:

$$
\begin{aligned}
& \mathbf{x}_{k+i+1 \mid k}=\mathbf{A} \mathbf{x}_{k+i \mid k}+\tilde{\mathbf{B}} \tilde{\mathbf{u}}_{k+i \mid k}+\tilde{\mathbf{B}}_{d} \mathbf{d}_{k+i \mid k}, \\
& \mathbb{P}\left[\mathbf{x}_{k+i+1 \mid k} \geq \mathbf{x}_{\min }\right] \geq 1-\frac{\delta_{x}}{2} \\
& \mathbb{P}\left[\mathbf{x}_{k+i+1 \mid k} \leq \mathbf{x}_{\max }\right] \geq 1-\frac{\delta_{x}}{2} \\
& \mathbb{P}\left[\mathbf{x}_{k+i+1 \mid k} \geq \mathbf{d}_{\text {net }, k+i+1 \mid k}\right] \geq 1-\delta_{s} \\
& \mathbf{u}_{\min } \leq \tilde{\mathbf{P}} \tilde{\mathbf{M}}_{1} \tilde{\mathbf{u}}_{k+i \mid k}+\tilde{\mathbf{P}} \tilde{\mathbf{M}}_{2} \mathbf{d}_{k+i \mid k} \leq \mathbf{u}_{\max }, \\
& \mathbf{d}_{\text {net }, k+i+1 \mid k}=\left|\mathbf{B}_{\text {out }}\left(\tilde{\mathbf{P}} \tilde{\mathbf{M}}_{1} \tilde{\mathbf{u}}_{k+i \mid k}+\tilde{\mathbf{P}} \tilde{\mathbf{M}}_{2} \mathbf{d}_{k+i \mid k}\right)+\mathbf{B}_{d} \mathbf{d}_{k+i+1 \mid k}\right|, \\
& \left(\mathbf{x}_{k \mid k}, \mathbf{d}_{k \mid k}\right)=\left(\mathbf{x}_{k}, \mathbf{d}_{k}\right)
\end{aligned}
$$

where $J_{E, k+i \mid k} \triangleq \sum_{i=0}^{H_{p}-1} \boldsymbol{\alpha}_{k+i \mid k}\left|\tilde{\mathbf{u}}_{k+i \mid k}\right|$ is the economic objective with $\boldsymbol{\alpha}_{k+i \mid k} \in \mathbb{R}^{n_{u}}$ being the cost of water production and transport, and $J_{\Delta U, k+i \mid k} \triangleq \sum_{i=0}^{H_{p}-1}\left\|\tilde{\mathbf{P}} \tilde{\mathbf{M}}_{1} \Delta \tilde{\mathbf{u}}_{k+i \mid k}+\tilde{\mathbf{P}} \tilde{\mathbf{M}}_{2} \Delta \mathbf{d}_{k+i \mid k}\right\|_{2}^{2}$ is the smoothness objective that penalises the control signal variations. Moreover, $\delta_{x}$ and $\delta_{s}$ are the accepted maximum risk for the state bounds and the safety constraint, respectively. The objectives are prioritised with scalar weights $\lambda_{1}, \lambda_{2}$. The service reliability goal (i.e., demand satisfaction) is enforced by the constraint $(36 \mathrm{e})$.

\section{3. $C C-M P C$ Reformulation}

In order to solve the above DWN control problem, a tractable safe approximation is derived following Section 3. The joint chance constraints (36c), (36d) and (36e), are transformed into deterministic equivalent constraints as

shown in Appendix B. Thus, Problem 2 for the case study is stated as follows for $i \in \mathbb{N}_{0}^{H_{p}-1}$ and $j \in \mathbb{N}_{1}^{n_{x}}$ : 


$$
J^{*} \triangleq \min _{\underline{\underline{\tilde{u}}}_{k}^{*}, \underline{\boldsymbol{\xi}}^{*}} \mathbb{E}\left[\lambda_{1} \sum_{i=0}^{H_{p}-1} J_{E, k+i \mid k}+\lambda_{2} \sum_{i=0}^{H_{p}-1} J_{\Delta U, k+i \mid k}+\lambda_{3} \sum_{i=0}^{H_{p}-1} J_{S, k+i+1 \mid k}\right],
$$

subject to:

$$
\begin{aligned}
& \overline{\mathbf{x}}_{k+i+1 \mid k}=\mathbf{A} \overline{\mathbf{x}}_{k+i \mid k}+\tilde{\mathbf{B}} \tilde{\mathbf{u}}_{k+i \mid k}+\tilde{\mathbf{B}}_{d} \overline{\mathbf{d}}_{k+i \mid k}, \\
& \overline{\mathbf{x}}_{(j), k+i+1 \mid k} \geq \mathbf{x}_{\min (j)}+\Phi^{-1}\left(1-\frac{\delta_{x}}{2 n_{x} H_{p}}\right) \Sigma_{\mathbf{x}_{(j)}, k+i+1 \mid k}^{1 / 2}, \\
& \overline{\mathbf{x}}_{(j), k+i+1 \mid k} \leq \mathbf{x}_{\max (j)}-\Phi^{-1}\left(1-\frac{\delta_{x}}{2 n_{x} H_{p}}\right) \Sigma_{\mathbf{x}_{(j)}, k+i+1 \mid k}^{1 / 2}, \\
& \overline{\mathbf{x}}_{(j), k+i+1 \mid k} \geq \overline{\mathbf{d}}_{\mathrm{net}(j), k+i+1 \mid k}+\Phi^{-1}\left(1-\frac{\delta_{s}}{n_{x} H_{p}}\right)\left\|\Sigma_{\mathbf{d}, k+i+1 \mid k}^{1 / 2} \tilde{\mathbf{B}}_{d(j)}^{\mathrm{T}}\right\|_{2}-\boldsymbol{\xi}_{k+i+1 \mid k},
\end{aligned}
$$

$$
\begin{aligned}
& \mathbf{u}_{\min } \leq \tilde{\mathbf{P}} \tilde{\mathbf{M}}_{1} \tilde{\mathbf{u}}_{k+i \mid k}+\tilde{\mathbf{P}} \tilde{\mathbf{M}}_{2} \overline{\mathbf{d}}_{k+i \mid k} \leq \mathbf{u}_{\max }, \\
& \overline{\mathbf{d}}_{\text {net }, k+i+1 \mid k}=\left|\mathbf{B}_{\text {out }}\left(\tilde{\mathbf{P}} \tilde{\mathbf{M}}_{1} \tilde{\mathbf{u}}_{k+i \mid k}+\tilde{\mathbf{P}} \tilde{\mathbf{M}}_{2} \overline{\mathbf{d}}_{k+i \mid k}\right)+\mathbf{B}_{d} \overline{\mathbf{d}}_{k+i+1 \mid k}\right|, \\
& \left(\overline{\mathbf{x}}_{k \mid k}, \overline{\mathbf{d}}_{k \mid k}\right)=\left(\mathbf{x}_{k}, \mathbf{d}_{k}\right) .
\end{aligned}
$$

Notice that (37a) includes a new objective $J_{S, k+i+1 \mid k} \triangleq \sum_{i=0}^{H_{p}-1}\left\|\boldsymbol{\xi}_{k+i+1 \mid k}\right\|_{2}^{2}$ that is related with the safety operational goal. This objective appears after relaxing the safety deterministic equivalent constraint (37e) with the slack decision variable $\boldsymbol{\xi} \in \mathbb{R}^{n_{x}}$ to guarantee feasibility of the optimisation problem and to allow the trading between safety, economic and smoothness objectives. Remark 5. After enforcing the chance constraints, some robust feasibility guarantees are obtained with an optimal back-off from the nominal deterministic constraints as a risk averse mechanism to face the non-stationary 
uncertainty involved in the prediction model of the MPC. The states are forced to move away from their limits before the disturbances have chance to cause constraint violation. The $\Phi^{-1}(\cdot)$ terms represent safety factors for each constraint, and especially in (37e), it denotes the optimal safety stock of storage tanks.

As it can be seen, the deterministic FHOP in (37) may be casted as a second-order cone programming problem. However, uncertainty in states is a function of the measured disturbances uncertainty only and is not a function of the decision variables of the optimisation problem. Therefore, the variance terms in each deterministic equivalent can be forecasted prior to the solution of the optimisation problem to include them as constants in the MPC formulation. This simplification results in a set of linear constraints and the optimisation remains as a quadratic programming (QP) problem, which can be efficiently solved.

\subsection{Demand Modelling}

Regular forecasting of a vast number of univariate time series is an essential task to develop proper controllers for the operational management of the DWN. The open-loop feed-forward uncertainty in the DWN can be modelled by the relationship between predicted states and predicted disturbances, see (B.2) and (B.3). In the dynamic model (1) of the DWN, randomness is directly described by the uncertainty of customer demands, which can be estimated from historical data. Figure 1 shows the histogram of a specific water demand node in the Barcelona DWN for the same time instant in different days during year 2007. It can be seen, in the envelope of the histogram, that the uncertain demand obeys a probabilistic distribution close 


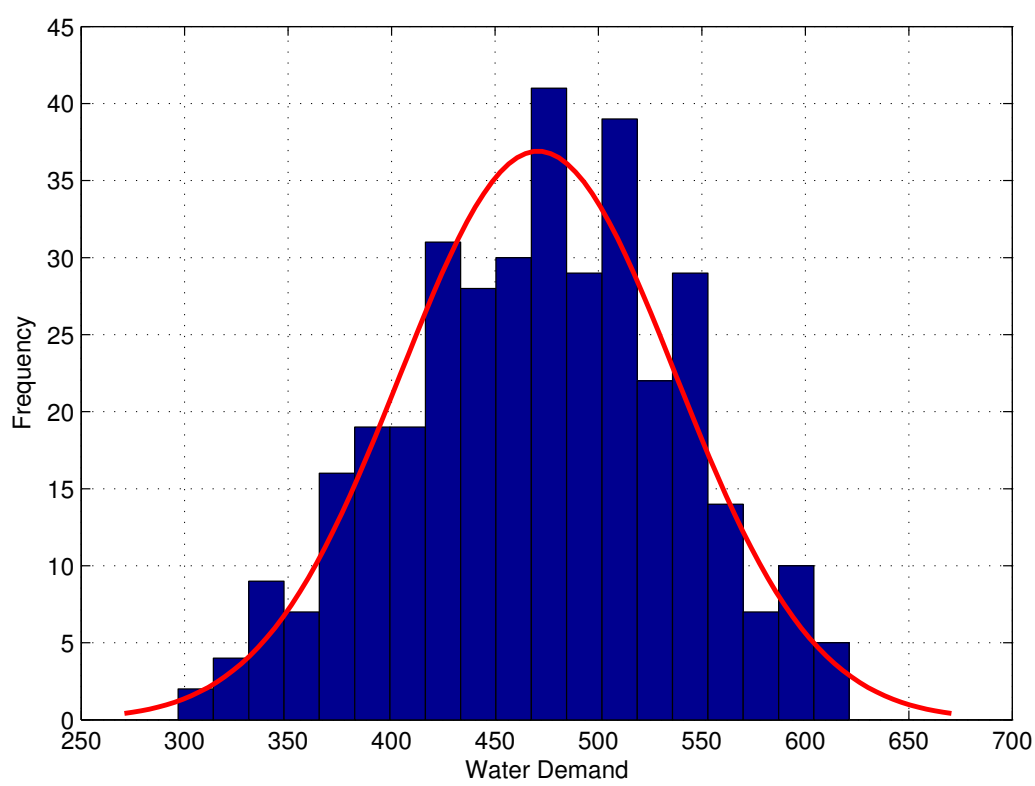

Fig. 1: Histogram of demand $c 176 B$ ARsud in the Barcelona DWN

to a Gaussian distribution (red curve). In addition, as shown in the last two plots of Figure 2 and discussed in [14], the demand pattern presents two seasonal cycles, one with a daily period and the other one with a weekly period.

In order to compute the vector of future disturbances and its stochastic properties required in the deterministic equivalent CC-MPC problem, this paper follows the modified exponential smoothing state-space framework developed in [38] for an automatic forecasting of complex seasonal time series (see Appendix C), such as the ones related to water demands in the Barcelona DWN. This framework extends traditional exponential smoothing models to accommodate multiple seasonality. The forecasting model is named BATS, which is an acronym for time-series models with Box-Cox transformation, 
Decomposition by BATS model

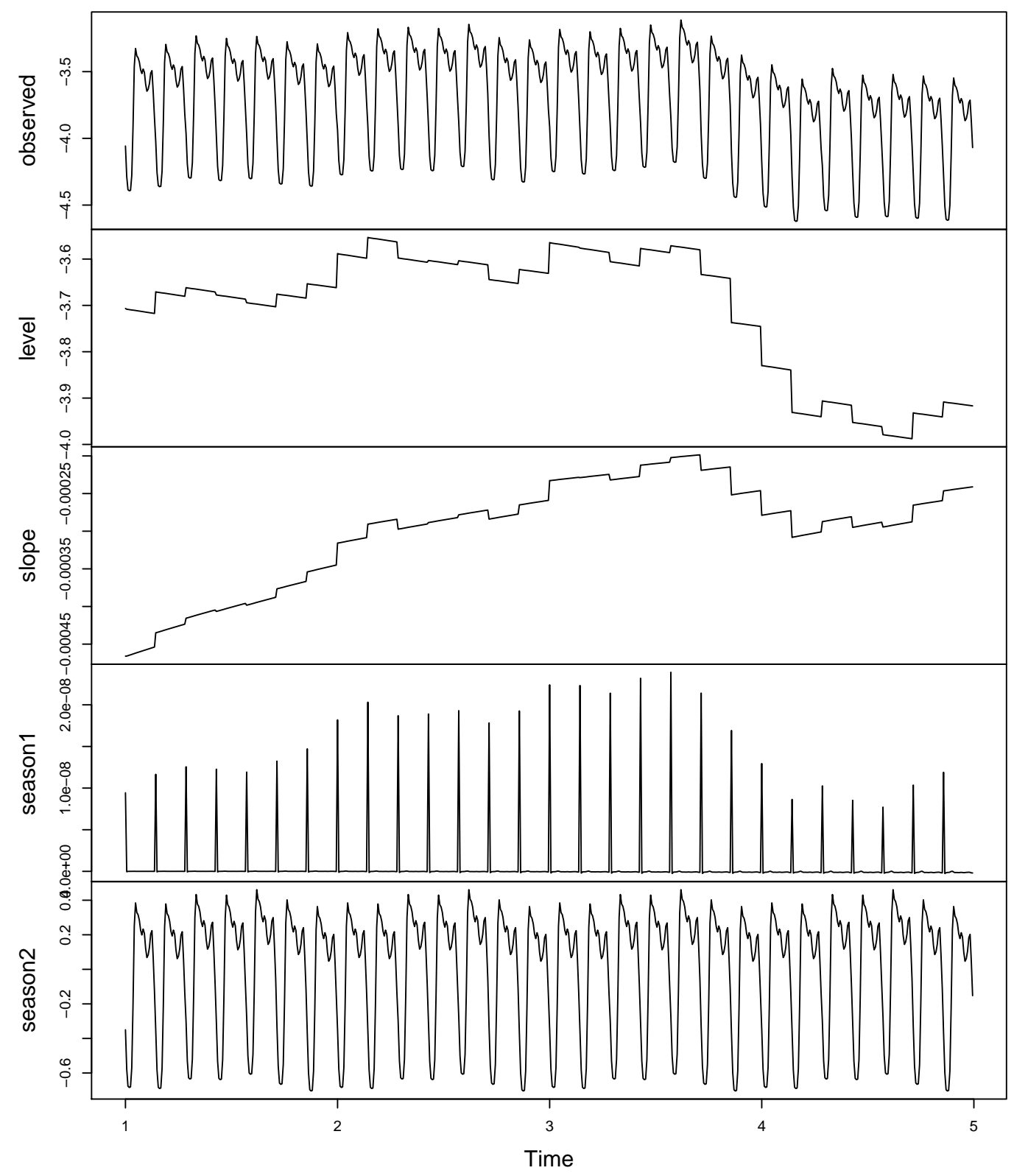

Fig. 2: Time series decomposition by BATS model for water demand forecasting in the Barcelona DWN 


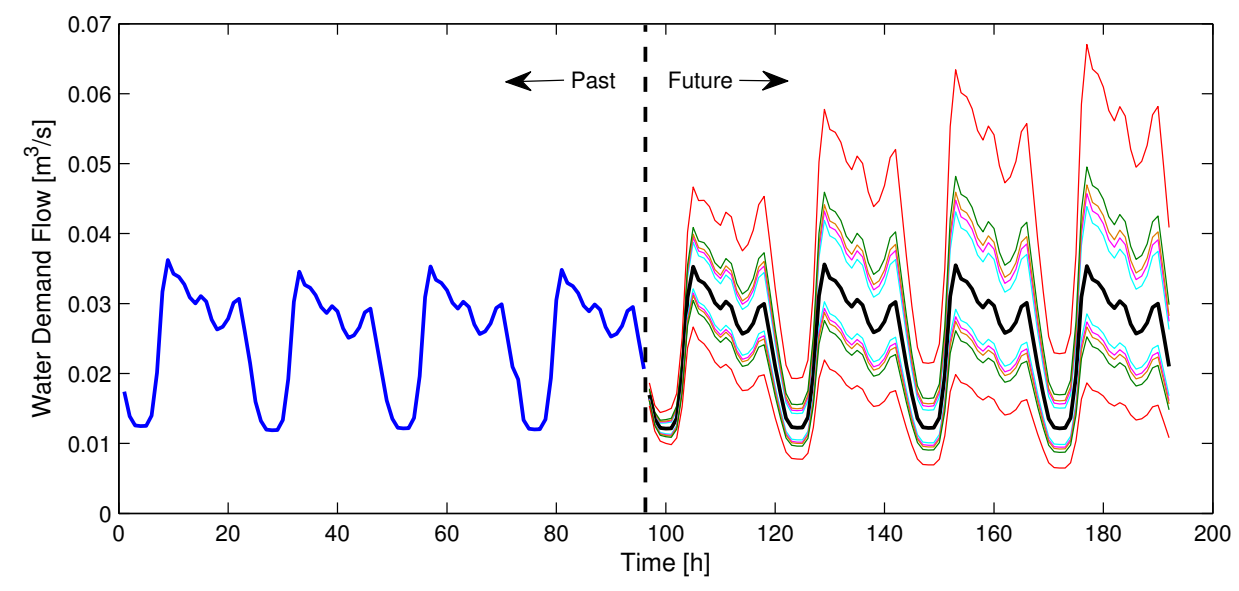

Fig. 3: Forecasting of water demand using a BATS model

ARMA errors, Trend and Seasonality components. Taking the $n_{d}$ time series of demands in the Barcelona DWN, and computing the BATS forecasting method presented in [38], it is possible to define the elements that complete the deterministic equivalent CC-MPC approach, i.e., the predicted mean and covariance. Figure 3 shows an open-loop forecast of a demand along a prediction horizon of four days, highlighting the effect of the propagated uncertainty. The thick line is the expectation of future demand, while the thin lines are the upper and lower bounds of the prediction interval for different confidence levels. For more details about the prediction of timeseries uncertainty description, the reader is referred to the aforementioned reference.

\subsection{Results}

This section presents the results of applying the CC-MPC approach (the deterministic equivalent) to the Barcelona DWN. The analysis has been carried out over a time period of four days (96 hours) with a sampling time 
of one hour. Demand scenarios correspond to real values reported between July $23^{\text {th }}$ and July $27^{\text {th }}, 2007$. Initial conditions, i.e., source capacities, initial volume of water at tanks and starting demands, are set a priori according to real data. The weights of the cost function (37a) are $\lambda_{1}=100, \lambda_{2}=0.005$, and $\lambda_{3}=10$. The tuning of these parameters has been chosen in a way that the objective with highest priority is the economic cost, which should be minimised while maintaining proper levels of safety volumes and control action smoothness. The prediction horizon is selected as $H_{p}=24 \mathrm{~h}$, due to the periodicity of the disturbances. The simulation has been carried out using the TOMLAB ${ }^{\circledR} 7.6$ optimisation package, and Matlab ${ }^{\circledR}$ R2010b (64 bits), running in a PC Intel ${ }^{\circledR}$ Core $^{\text {TM }}$ E8600 at $3.33 \mathrm{GHz}$ with $8 \mathrm{~GB}$ of RAM.

In order to assess the control enhancements developed in this paper, two control strategies have been simulated:

- CE-MPC: It is a (certainty equivalent) perturbed deterministic MPC that assumes predictions of demands as certain. This control strategy allows the volume of water in tanks to decrease until the predicted volume of future net demands, which is set as a hard constraint but ignoring the influence of uncertainty.

- CC-MPC: It is the chance-constrained approach presented in this paper, which considers and propagates the uncertainty of forecast demands explicitly in the MPC design and, as a consequence, involves a robust handling of constraints. To analyse the effect of the risk level $\left(\delta_{x}\right)$ in this CC-MPC strategy, different scenarios have been simulated for acceptable joint risks of $50 \%, 40 \%, 30 \%, 20 \%, 10 \%, 5 \%$ and $1 \%$. 
Table 1 presents the numeric assessment of the aforementioned controllers through different key performance indicators (KPIs), which are defined below:

$$
\begin{aligned}
& \mathrm{KPI}_{E} \triangleq \frac{1}{n_{s}} \sum_{k=1}^{n_{s}} \boldsymbol{\alpha}_{k}^{\mathrm{T}}\left|\mathbf{u}_{k}\right| \Delta t, \\
& \mathrm{KPI}_{\Delta U} \triangleq \frac{1}{n_{s}} \sum_{i=1}^{n_{u}} \sum_{k=1}^{n_{s}}\left(\Delta u_{(i), k}\right)^{2}, \\
& \mathrm{KPI}_{S} \triangleq \sum_{i=1}^{n_{x}} \sum_{k=1}^{n_{s}} \max \left\{0, x_{\mathrm{s}(i), k}-x_{(i), k}\right\}, \\
& \mathrm{KPI}_{D} \triangleq \sum_{i=1}^{n_{x}} \sum_{k=1}^{n_{s}} \max \left\{0, d_{\mathrm{net}(i), k}-x_{(i), k}\right\}, \\
& \mathrm{KPI}_{R} \triangleq \frac{\sum_{i=1}^{n_{x}} \sum_{k=1}^{n_{s}} x_{\mathrm{s}(i), k}}{\sum_{i=1}^{n_{x}} \sum_{k=1}^{n_{s}} x_{(i), k}} \times 100 \%, \\
& \mathrm{KPI}_{O} \triangleq t_{\mathrm{opt}, k},
\end{aligned}
$$

where $\mathrm{KPI}_{E}$ is the average economic performance of the DWN operation, $\mathrm{KPI}_{\Delta U}$ measures the smoothness of the control actions, $\mathrm{KPI}_{S}$ is the amount of water used from safety stocks, $\mathrm{KPI}_{D}$ is the volume of water demand that is not satisfied over the simulation period, $\mathrm{KPI}_{R}$ is the average percentage of safety volume that is contained in the real water volume, and $\mathrm{KPI}_{O}$ determines the difficulty to solve the optimisation tasks involved in each strategy accounting $t_{\mathrm{opt}, k}$ as the average time that takes to solve the FHOP.

The CE-MPC has been tuned with a safety stock for each tank equal to its net exogenous demand. Therefore, the $\mathrm{KPI}_{S}$ results equal to the $\mathrm{KPI}_{D}$ as should be expected given their definitions. Regarding the comparison of the $\mathrm{KPI}_{S}$ between the CE-MPC and the CC-MPC, the results present greater values for the CC-MPC cases. This trend is also an expected behaviour 
Table 1: Comparison of the MPC strategies applied to the Barcelona DWN

\begin{tabular}{lcccccc}
\hline Controller & $\mathrm{KPI}_{E}$ & $\mathrm{KPI}_{\Delta U}$ & $\mathrm{KPI}_{S}$ & $\mathrm{KPI}_{D}$ & $\mathrm{KPI}_{R}$ & $\mathrm{KPI}_{O}$ \\
\hline $\mathrm{CE}-\mathrm{MPC}$ & 2297.02 & 2.3586 & 3.8886 & 3.8886 & 19.41 & 4.82 \\
\hline $\mathrm{CC}-\mathrm{MPC}_{@ 50 \%}$ & 2486.40 & 1.0747 & 695.54 & 0 & 27.79 & 4.72 \\
\hline $\mathrm{CC}-\mathrm{MPC}_{@ 40 \%}$ & 2487.77 & 1.0767 & 750.06 & 0 & 27.86 & 4.83 \\
\hline $\mathrm{CC}-\mathrm{MPC}_{@ 30 \%}$ & 2489.31 & 1.0795 & 819.82 & 0 & 27.95 & 4.79 \\
\hline $\mathrm{CC}-\mathrm{MPC}_{@ 20 \%}$ & 2491.61 & 1.0835 & 920.36 & 0 & 28.07 & 4.71 \\
\hline $\mathrm{CC}-\mathrm{MPC}_{@ 10 \%}$ & 2496.23 & 1.0964 & 1101.7 & 0 & 28.18 & 4.70 \\
\hline $\mathrm{CC}-\mathrm{MPC}_{@ 5 \%}$ & 2500.52 & 1.1012 & 1298.9 & 0 & 28.18 & 4.89 \\
\hline $\mathrm{CC}-\mathrm{MPC}_{@ 1 \%}$ & 2509.89 & 1.1131 & 1759.4 & 0 & 28.43 & 4.86 \\
\hline
\end{tabular}

given that reducing the risk probability generates a larger back-off of the demand satisfaction constraint, i.e., more safety stock is stored to address demand uncertainty. This latter fact, in addition with the tuning of the multi-objective cost function, leads to higher $\mathrm{KPI}_{S}$ (but lower or null $\mathrm{KPI}_{D}$ ) if so is required by the real demand scenario in order to guarantee a service level. It can be observed that the CE-MPC is the cheapest control strategy (lower $\mathrm{KPI}_{E}$ ) but the less reliable one given that the certainty equivalence assumption leads to unsatisfying demands (higher $\mathrm{KPI}_{D}$ ), especially when the water volume in the tank is close to the expected demand. Thus, the CE-MPC performance represents a strategy for the supply of drinking water with a higher risk of failure. The different CC-MPC scenarios (those of varying the risk acceptability level) have shown that reliability and economic 
Table 2: Comparison of daily average economic costs of MPC strategies

\begin{tabular}{lccc}
\hline Controller & $\begin{array}{c}\text { Water Cost } \\
(\text { e.u./day })\end{array}$ & $\begin{array}{c}\text { Electric Cost } \\
\text { (e.u./day) }\end{array}$ & $\begin{array}{c}\text { Daily Average Cost } \\
\text { (e.u./day) }\end{array}$ \\
\hline CE-MPC & 2301.54 & 2719.53 & 5021.07 \\
\hline CC-MPC $_{9} \%$ & 2298.03 & 2851.47 & 5149.50 \\
\hline
\end{tabular}

e.u.: economic units.

performance are conflicting objectives which have to reach a trade-off, i.e., the inclusion of safety mechanisms in the controller increases the reliability of the DWN in terms of demand satisfaction (see Figure 4), but also the economic cost of its operation. The main advantage of the CC-MPC is its formal methodology that leads to obtain optimal dynamic constraints that tackle uncertainties with a minimum cost to achieve also a global service level of the DWN. Table 1 shows a smooth degradation of the economic performance under the CC-MPC when varying the risk within a wide range of acceptability levels. Therefore, the CC-MPC approach addressed in this paper is a suitable mean to compute the proper amount of safety and the proper control actions to assure a desired service level. Notice that the computational burden $\left(\mathrm{KPI}_{O}\right)$ of the $\mathrm{CC}-\mathrm{MPC}$ is similar to the $\mathrm{CE}-\mathrm{MPC}$ given that the complexity of the FHOP is not altered, i.e., the number of constraints and decision variables remain the same. The only extra load that might be added is the computation of the variance of the disturbances propagated in the prediction horizon. Consequently, the CC-MPC approach is suitable for real-time control (RTC) of the Barcelona DWN. 


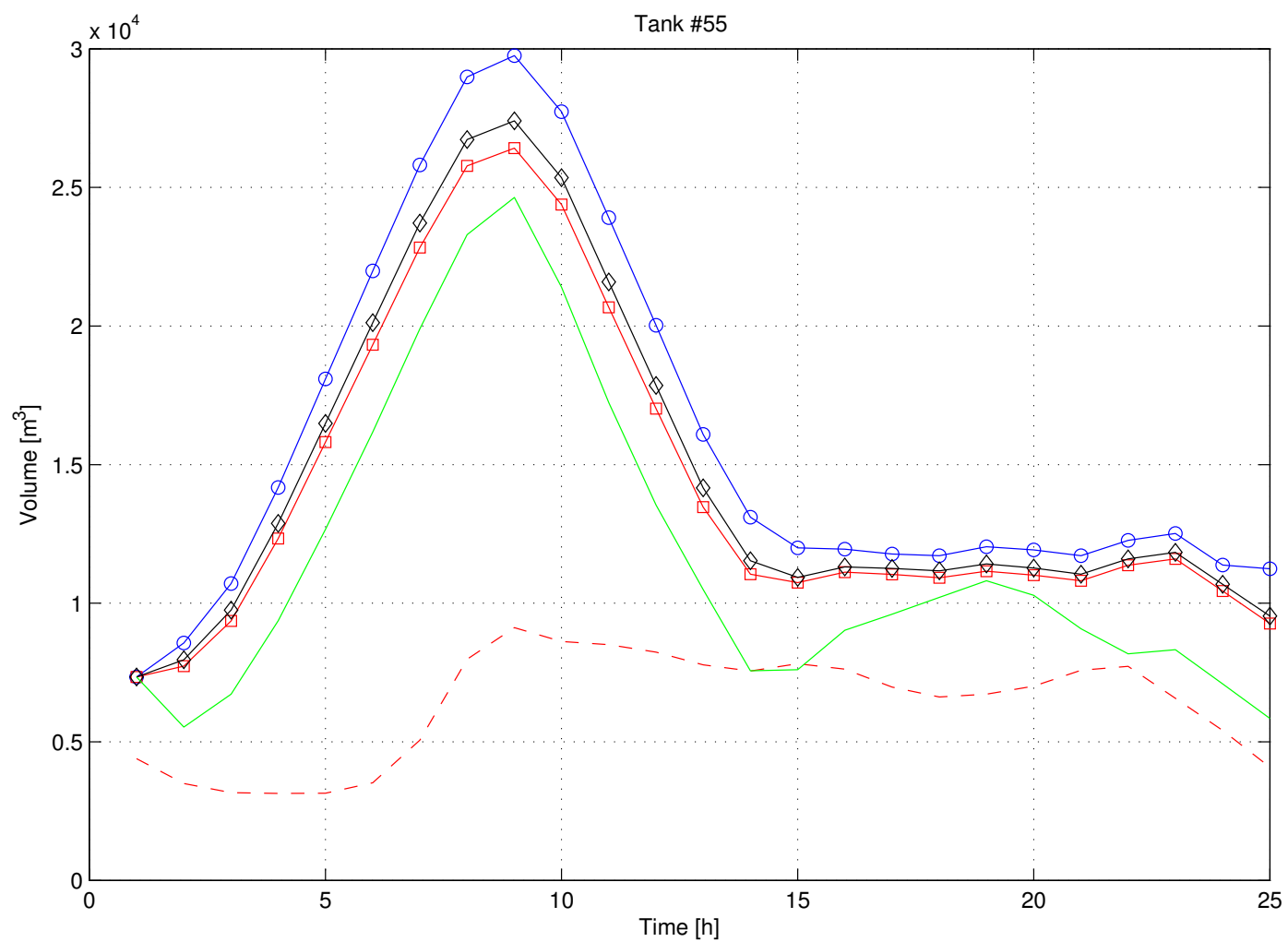

Fig. 4: Comparison of the robustness in the management of water storage in a sample of tanks of the Barcelona DWN. (blue circle) $\mathrm{CCMPC}_{1 \%}$, (black diamond) $\mathrm{CCMPC}_{20 \%}$, (red square) $\mathrm{CCMPC}_{50 \%}$, (solid green) CEMPC, (dashed red) Net demand 
Table 3: Conservatism of the Deterministic Equivalent CC-MPC

\begin{tabular}{llll}
\hline Joint Chance Constraint & $\begin{array}{c}\text { Number of Individual } \\
\text { Constraints }\end{array}$ & Joint Risk & $\begin{array}{c}\text { Conservatism } \\
\text { of Approximation }\end{array}$ \\
\hline & & 0.001 & $4.9967 \times 10^{-7}$ \\
& 0.01 & $4.9817 \times 10^{-5}$ \\
State Hard Bounds & 0.03 & $4.4539 \times 10^{-4}$ \\
& & 0.05 & $1.2290 \times 10^{-3}$ \\
& & 0.1 & $4.8359 \times 10^{-3}$ \\
\hline \multirow{2}{*}{1512} & 0.001 & $4.9950 \times 10^{-7}$ \\
& & 0.01 & $4.9801 \times 10^{-5}$ \\
& & 0.05 & $4.4524 \times 10^{-4}$ \\
& & 0.1 & $4.8344 \times 10^{-3}$ \\
\hline
\end{tabular}

Table 2 discloses details of the average production and operational costs related to each strategy. Comparing the CE-MPC controller with the CC-MPC $@ 5 \%$ controller (requiring a reliability of 95\%), it can be noticed that the dynamic safety stocks resulting within the stochastic approach might lead to an increase of the operational cost, especially in the electric cost, mainly due to the extra amount of water that is needed to be moved through the network and allocated in tanks to guarantee that the water supply will be feasible with a certain probability for future disturbance realisations.

The conservatism of reformulating the stochastic Problem 1 into the tractable deterministic equivalent in (37) is shown in Table 3 for the chance constraints (36c), (36d) and (36e), with different levels of maximum joint risk. 
It can be observed that conservatism increases when the risk level increases but remains almost constant despite the variation of the number of individual constraints. Hence, the goodness of the approximation using Boole's inequality is not affected, neither by the number of decision variables, nor by the prediction horizon. Therefore, the addressed approach is advantageous to be applied to any other DWNs or general flow networks.

\section{Conclusions}

This paper addresses a CC-MPC strategy that explicitly incorporates the uncertainty information into a stochastic optimisation problem by replacing hard constraints of states with joint probabilistic constraints and by replacing the nominal cost function with its expected value in the MPC formulation. Thus, the resulting decisions are robust and ensure the optimisation of the dynamic performance and the reliability of remaining feasible. The analytical approximation of joint chance constraints based on their decomposition into individual chance constraints, these latter bounded by means of Boole's inequality, has shown to be suitable for large DWNs regarding that the conservatism involved is not affected neither by the number of the inequal-

ities nor the prediction horizon of the MPC. The level of resultant back-off is variable and depends on the volatility of the forecast demand at each prediction step. In this CC-MPC approach, a BATS time-series model of the disturbance stochastic properties was used in parallel with the MPC model. The CC-MPC presented here focuses on robust performance of DWNs, where additive disturbances (unbounded and stationary or non-stationary) constitute the main uncertainty. The CC-MPC strategy enhances the robustness 
of the baseline CE-MPC by performing a dynamic handling of constraints in a systematic way instead of using heuristic fixed safety volumes as in the CE-MPC. The fact of non-bounded disturbances in the system precludes the guarantee of robust feasibility. Hence, the approach proposed in this paper is based on a service level guarantee and a probabilistic feasibility. The casestudy shows that the CC-MPC is suitable for the operational guidance of DWNs due to its robustness, flexibility, modest computational requirements, and ability to include risk considerations directly in the decision-making process. Even when the CC-MPC increased the operational costs by around $2.5 \%$, it allowed to improve service reliability by more than $90 \%$. Future research will be directed to incorporate parametric uncertainty and unmeasured disturbances in the model. Moreover, it is the interest of the authors to extend the results and develop decentralised/distributed stochastic MPC controllers for large-scale complex flow networks.

\section{Appendix A. DWN variables reduction}

The reduction of the model is based on the following assumption.

Assumption 3. There are more variables than algebraic equations, i.e., $n_{q}<n_{u}$. The matrix $\mathbf{E}_{u}$ in (1b) has maximal rank, i.e. $\operatorname{rank}\left(\mathbf{E}_{u}\right)=n_{q}$, and it can be expressed in a reduced staggered form by using Gauss-Jordan elimination.

Consider (1b) in the following form:

$$
\left[\begin{array}{ll}
\mathbf{E}_{u} & \mathbf{E}_{d}
\end{array}\right]\left[\begin{array}{l}
\mathbf{u} \\
\mathbf{d}
\end{array}\right]=\mathbf{0} .
$$


Assumption 3 guarantees that there exists a permutation $\tilde{\mathbf{P}}$ of the first $n_{u}$ variables in (A.1) such that

$$
\mathbf{E}_{u} \tilde{\mathbf{P}}=\left[\begin{array}{ll}
\mathbf{I}_{n_{q}} & \mathbf{M}_{1}
\end{array}\right], \quad \mathbf{M}_{1} \in \mathbb{R}^{n_{q} \times\left(n_{u}-n_{q}\right)},
$$

and

$$
\left[\begin{array}{ll}
\mathbf{E}_{u} & \mathbf{E}_{d}
\end{array}\right] \mathbf{P}=\left[\begin{array}{lll}
\mathbf{I}_{n_{q}} & \mathbf{M}_{1} & \mathbf{E}_{d}
\end{array}\right], \quad \mathbf{E}_{d} \in \mathbb{R}^{n_{q} \times n_{d}}
$$

where

$$
\mathbf{P}=\left[\begin{array}{cc}
\tilde{\mathbf{P}} & \mathbf{0} \\
\mathbf{0} & \mathbf{I}_{n_{d}}
\end{array}\right]
$$

Then, it is possible to state that

$$
\left[\begin{array}{ll}
\mathbf{E}_{u} & \mathbf{E}_{d}
\end{array}\right]\left[\begin{array}{l}
\mathbf{u} \\
\mathbf{d}
\end{array}\right]=\mathbf{0} \Longleftrightarrow\left[\begin{array}{ll}
\mathbf{E}_{u} & \mathbf{E}_{d}
\end{array}\right] \mathbf{P} \mathbf{P}^{\mathrm{T}}\left[\begin{array}{l}
\mathbf{u} \\
\mathbf{d}
\end{array}\right]=\mathbf{0} .
$$

Defining

$$
\left[\begin{array}{l}
\mathbf{v} \\
\mathbf{d}
\end{array}\right]=\mathbf{P}^{\mathrm{T}}\left[\begin{array}{l}
\mathbf{u} \\
\mathbf{d}
\end{array}\right]=\left[\begin{array}{c}
\tilde{\mathbf{P}}^{\mathrm{T}} \mathbf{u} \\
\mathbf{d}
\end{array}\right]
$$

where

$$
\mathbf{v}=\left[\begin{array}{c}
\overline{\mathbf{u}} \\
\tilde{\mathbf{u}}
\end{array}\right], \quad \overline{\mathbf{u}} \in \mathbb{R}^{n_{q}}, \quad \tilde{\mathbf{u}} \in \mathbb{R}^{\left(n_{u}-n_{q}\right)},
$$

then it holds

$$
\left[\begin{array}{lll}
\mathbf{I}_{n_{q}} & \mathbf{M}_{1} & \mathbf{E}_{d}
\end{array}\right]\left[\begin{array}{c}
\overline{\mathbf{u}} \\
\tilde{\mathbf{u}} \\
\mathbf{d}
\end{array}\right]=\mathbf{0} \Longrightarrow \overline{\mathbf{u}}=-\mathbf{M}_{1} \tilde{\mathbf{u}}-\mathbf{E}_{d} \mathbf{d},
$$


and

$$
\begin{aligned}
\mathbf{v} & =\left[\begin{array}{c}
\overline{\mathbf{u}} \\
\tilde{\mathbf{u}}
\end{array}\right]=\left[\begin{array}{c}
-\mathbf{M}_{1} \tilde{\mathbf{u}}-\mathbf{E}_{d} \mathbf{d} \\
\tilde{\mathbf{u}}
\end{array}\right] \\
& =\left[\begin{array}{c}
-\mathbf{M}_{1} \\
\mathbf{I}_{\left(n_{u}-n_{q}\right)}
\end{array}\right] \tilde{\mathbf{u}}+\left[\begin{array}{c}
-\mathbf{E}_{d} \\
\mathbf{0}_{n_{d}}
\end{array}\right] \mathbf{d} .
\end{aligned}
$$

Finally, being $\mathbf{P}$ a permutation matrix and therefore an orthogonal matrix, i.e., $\mathbf{P}^{-1}=\mathbf{P}^{\mathrm{T}}$, and from $\tilde{\mathbf{P}}^{\mathrm{T}} \mathbf{u}=\mathbf{v}$, the control parametrisation is as follows:

$$
\mathbf{u}=\tilde{\mathbf{P}} \underbrace{\left[\begin{array}{c}
-\mathbf{M}_{1} \\
\mathbf{I}_{\left(n_{u}-n_{q}\right)}
\end{array}\right]}_{\tilde{\mathbf{M}}_{1}} \tilde{\mathbf{u}}+\tilde{\mathbf{P}} \underbrace{\left[\begin{array}{c}
-\mathbf{E}_{d} \\
\mathbf{0}_{n_{d}}
\end{array}\right]}_{\tilde{\mathbf{M}}_{2}} \mathbf{d} .
$$

Replacing (A.10) in (1), the system can be then modelled with the following difference equation:

$$
\mathbf{x}_{k+1}=\mathbf{A} \mathbf{x}_{k}+\tilde{\mathbf{B}} \tilde{\mathbf{u}}_{k}+\tilde{\mathbf{B}}_{d} \mathbf{d}_{k}
$$

where

$$
\tilde{\mathbf{B}}=\mathbf{B} \tilde{\mathbf{P}} \tilde{\mathbf{M}}_{1}, \quad \tilde{\mathbf{B}}_{d}=\mathbf{B} \tilde{\mathbf{P}} \tilde{\mathbf{M}}_{2}+\mathbf{B}_{d}
$$

In the same way, constraint (3b) is transformed taking into account the control parametrisation in (A.10). Therefore, the set of restricted input constraints is defined as

$$
\tilde{\mathcal{U}}_{k} \triangleq\left\{\tilde{\mathbf{u}}_{k} \in \mathbb{R}^{n_{\tilde{u}}} \mid \mathbf{F} \tilde{\mathbf{P}} \tilde{\mathbf{M}}_{1} \tilde{\mathbf{u}}_{k} \leq \mathbf{f}-\mathbf{F} \tilde{\mathbf{P}} \tilde{\mathbf{M}}_{2} \mathbf{d}_{k}\right\} \subset \mathbb{R}^{n_{\tilde{u}}}, \quad \forall k
$$




\section{Appendix B. DWN chance constraints convex approximation}

Below is derived the deterministic equivalents of the individual chance constraints that approximate the joint chance constraints in (36).

Lower Bound of States: The robust counterpart of the set of individual chance constraints that approximates the joint constraint (36c) is derived as follows:

$$
\begin{aligned}
\forall i \in \mathbb{N}_{0}^{H_{p}-1} \wedge & \forall j \in \mathbb{N}_{1}^{n_{x}}, \\
& \mathbb{P}\left[\mathbf{x}_{(j), k+i+1 \mid k} \geq \mathbf{x}_{\min (j)}\right] \geq 1-\frac{\delta_{x}}{2 n_{x} H_{p}} \\
\Leftrightarrow & \mathbb{P}\left[\mathbf{x}_{(j), k+i+1 \mid k}<\mathbf{x}_{\min (j)}\right] \leq \frac{\delta_{x}}{2 n_{x} H_{p}} \\
\Leftrightarrow & \mathbb{P}\left[\frac{\mathbf{x}_{(j), k+i+1 \mid k}-\overline{\mathbf{x}}_{(j), k+i+1 \mid k}}{\Sigma_{\mathbf{x}_{(j)}, k+i+1 \mid k}^{1 / 2}}<\frac{\mathbf{x}_{\min (j)}-\overline{\mathbf{x}}_{(j), k+i+1 \mid k}}{\sum_{\mathbf{x}_{(j)}, k+i+1 \mid k}^{1 / 2}}\right] \leq \frac{\delta_{x}}{2 n_{x} H_{p}} \\
\Leftrightarrow & \Phi\left(\frac{\mathbf{x}_{\min (j)}-\overline{\mathbf{x}}_{(j), k+i+1 \mid k}}{\Sigma_{\mathbf{x}_{(j)}^{1 / 2}, k+i+1 \mid k}^{1}}\right) \leq \frac{\delta_{x}}{2 n_{x} H_{p}}, \\
\Leftrightarrow & \overline{\mathbf{x}}_{(j), k+i+1 \mid k} \geq \mathbf{x}_{\min (j)}-\Phi^{-1}\left(\frac{\delta_{x}}{2 n_{x} H_{p}}\right) \Sigma_{\mathbf{x}_{(j)}, k+i+1 \mid k}^{1 / 2} .
\end{aligned}
$$

The mean and variance of $\mathbf{x}$ are computed over the random variable $\mathbf{d}$, as follows:

$$
\begin{aligned}
\overline{\mathbf{x}}_{(j), k+i+1 \mid k} & =\mathbf{A}_{(j)} \overline{\mathbf{x}}_{k+i \mid k}+\tilde{\mathbf{B}}_{(j)} \tilde{\mathbf{u}}_{k+i \mid k}+\tilde{\mathbf{B}}_{d(j)} \overline{\mathbf{d}}_{k+i \mid k}, \\
\Sigma_{\mathbf{x}_{(j)}, k+i+1 \mid k} & =\mathbf{A}_{(j)} \Sigma_{\mathbf{x}, k+i \mid k} \mathbf{A}_{(j)}^{\mathrm{T}}+\tilde{\mathbf{B}}_{d(j)} \Sigma_{\mathbf{d}, k+i \mid k} \tilde{\mathbf{B}}_{d(j)}^{\mathrm{T}}, .
\end{aligned}
$$

The symmetry of the normal distribution allows to consider the equality $-\Phi^{-1}(p)=\Phi(1-p)$ for any probability level $p \in(0,1)$. In this way, the equivalent of (36c) can be finally expressed, $\forall i \in \mathbb{N}_{0}^{H_{p}-1}$ and $\forall j \in \mathbb{N}_{1}^{n_{x}}$, by 
the following single constraints:

$$
\overline{\mathbf{x}}_{(j), k+i+1 \mid k} \geq \mathbf{x}_{\min (j)}+\Phi^{-1}\left(1-\frac{\delta_{x}}{2 n_{x} H_{p}}\right) \Sigma_{\mathbf{x}_{(j)}, k+i+1 \mid k}^{1 / 2} .
$$

Upper Bound of States: The same procedure used to derive the lower bound of states yields the robust counterpart of (36d), which is expressed, $\forall i \in \mathbb{N}_{0}^{H_{p}-1}$ and $\forall j \in \mathbb{N}_{1}^{n_{x}}$, as

$$
\overline{\mathbf{x}}_{(j), k+i+1 \mid k} \leq \mathbf{x}_{\max (j)}-\Phi^{-1}\left(1-\frac{\delta_{x}}{2 n_{x} H_{p}}\right) \Sigma_{\mathbf{x}_{(j)}, k+i+1 \mid k}^{1 / 2} .
$$

Safety Constraint of States: In the operational constraint (36e), both sides of the inequality $\mathbf{x}_{k+i+1 \mid k} \geq \mathbf{d}_{\text {net, }, k+i+1 \mid k}$ contain random variables. This fact could complicate the definition of a linear deterministic equivalent for the probabilistic constraint, unless appropriate assumptions are made. As it can be seen in (36b) and (36g), the uncertainty in variables $\mathbf{x}_{k+i+1 \mid k}$ and $\mathbf{d}_{\text {net }, k+i+1 \mid k}$ is directly associated with the stochastic variable in common: the forecasted demand $\mathbf{d}$, which appears in the definition of both sides of the aforementioned inequality, but with a difference of one time instant between each side. Therefore, taking into account that a disturbance prediction model (e.g., time-series model) may allow to estimate the cumulative uncertainty for a multiple-step forecasting process, it can be assumed that the uncertainty of $\mathbf{d}_{k+i \mid k}$ is already considered in the uncertainty of $\mathbf{d}_{k+i+1 \mid k}$. This assumption avoids overestimation of uncertainty and aims to reduce conservatism in the controller performance. 
The deterministic equivalent is derived as follows:

$$
\begin{aligned}
& \forall i \in \mathbb{N}_{0}^{H_{p}-1} \wedge \forall j \in \mathbb{N}_{1}^{n_{x}}, \quad \mathbb{P}\left[\mathbf{x}_{(j), k+i+1 \mid k} \geq \mathbf{d}_{\text {net }(j), k+i+1 \mid k}\right] \geq 1-\frac{\delta_{s}}{n_{x} H_{p}} \\
& \Leftrightarrow \quad \mathbb{P}\left[\mathbf{d}_{\text {net }(j), k+i+1 \mid k}-\mathbf{x}_{(j), k+i+1 \mid k} \leq \mathbf{0}\right] \geq 1-\frac{\delta_{s}}{n_{x} H_{p}} \\
& \Leftrightarrow \quad \Phi\left(\frac{-\mathbb{E}\left[\mathbf{d}_{\text {net }(j), k+i+1 \mid k}-\mathbf{x}_{(j), k+i+1 \mid k}\right]}{\left.\Sigma_{\mathbf{d}_{\text {net }(j)}^{1 / 2}, k+i+1 \mid k}^{1 / 2}\right) \geq 1-\frac{\delta_{s}}{n_{x} H_{p}}}\right. \\
& \Leftrightarrow \quad \overline{\mathbf{x}}_{(j), k+i+1 \mid k} \geq \overline{\mathbf{d}}_{\text {net }(j), k+i+1 \mid k}+\Phi^{-1}\left(1-\frac{\delta_{s}}{n_{x} H_{p}}\right) \Sigma_{\mathbf{d}_{\text {net }(j)}, k+i+1 \mid k} .
\end{aligned}
$$

\section{Appendix C. BATS Modelling of Water Demand}

Demand time series model: Let $d_{k}, k \in \mathbb{N}_{+}$, denote an observed time series of any water demand, and $d_{k}^{(\omega)}$ the Box-Cox transformed observed value at time $k$ with the parameter $\omega$. The transformed series $d_{k}^{(\omega)}$, is then decomposed into an irregular component $h_{k}$, a level component $l_{k}$, a growth component $b_{k}$ and possible seasonal components $s_{k}^{(i)}$ with seasonal frequencies $m_{i}$, for $i=1, \ldots, P$, where $P$ is the total number of seasonal patterns in the series. In order to allow for possible dampening of the trend, a damping parameter $\phi$ is included. The irregular component $h_{k}$ is described by an $\operatorname{ARMA}(p, q)$ process with parameters $\varphi_{i}$ for $i=1, \ldots, p$ and $\theta_{i}$ for $i=1, \ldots, q$, and an error term $\varepsilon_{k}$ which is assumed to be a Gaussian white noise process with zero mean and constant variance $\sigma^{2}$. The smoothing parameters, given by $\alpha_{d}, \beta_{d}, \gamma_{d, i}$ for $i=1, \ldots, P$, determine the extent of the

effect of the irregular component on the states $l_{k}, b_{k}, s_{k}^{(i)}$ respectively. The 
equations for the models are

$$
\begin{array}{ccc}
d_{k}^{(\omega)}= & \left\{\begin{array}{cc}
\frac{d_{k}^{(\omega)}-1}{\omega}, & \omega \neq 0, \\
\log \left(d_{k}\right), & \omega=0,
\end{array}\right. \\
d_{k}^{(\omega)}= & l_{k-1}+\phi b_{k-1}+\sum_{i=1}^{P} s_{k-m_{i}}^{(i)}+h_{k}, \\
l_{k}= & l_{k-1}+\phi b_{k-1}+\alpha_{d} h_{k}, \\
b_{k}= & \phi b_{k-1}+\beta_{d} h_{k}, \\
s_{k}^{(i)}= & s_{k-m_{i}}^{(i)}+\gamma_{d, i} h_{k}, \\
h_{k}= & \sum_{i=1}^{p} \varphi_{i} h_{k-i}+\sum_{i=1}^{q} \theta_{i} \varepsilon_{k-i}+\varepsilon_{k} .
\end{array}
$$

The above model receives the notation $\operatorname{BATS}\left(p, q, m_{1}, m_{2}, \ldots, m_{P}\right)$ and it can be expressed in the following state-space form:

$$
\begin{aligned}
& d_{k}^{(\omega)}=\mathbf{w}^{\mathrm{T}} \mathbf{x}_{\mathrm{d}, k-1}+\varepsilon_{k}, \\
& \mathbf{x}_{\mathrm{d}, k}=\mathbf{F} \mathbf{x}_{d, k-1}+\mathbf{g} \varepsilon_{k},
\end{aligned}
$$

where $\mathbf{w}^{\mathrm{T}}$ is a row vector, $\mathbf{g}$ is a column vector, $\mathbf{F}$ is a square matrix and $\mathbf{x}_{\mathrm{d}, k}$ is the unobserved demand state vector at time $k$. The details on how these vectors and matrices are defined can be found in [38].

Demand uncertainty propagation: Let $\vartheta$ be a vector of all parameters to be estimated in the model (C.1-C.6), including the smoothing parameters and the Box-Cox parameter. Moreover, let $k$ be the actual length of a water demand time series, $n$ be the length of the desired demand forecast horizon, and $d_{k+n \mid k} \triangleq\left\{d_{k+n} \mid \mathbf{x}_{d, k}, \boldsymbol{\vartheta}\right\}$ be a random variable denoting future values of a demand series given the model, its calibrated parameters and the demand state vector at the last observation $\mathbf{x}_{\mathrm{d}, k}$. A Gaussian assumption for the errors implies that $d_{k+n \mid k}^{(\omega)}$ is also normally distributed, with mean $\bar{d}_{k+n \mid k}^{(\omega)}$ and 
variance $\Sigma_{d, k+n \mid k}^{(\omega)}$ given by

$$
\begin{aligned}
\bar{d}_{k+n \mid k}^{(\omega)} & =\mathbf{w}^{\mathrm{T}} \mathbf{F}^{n-1} \mathbf{x}_{\mathrm{d}, k}, \\
\Sigma_{d, k+n \mid k}^{(\omega)} & =\left\{\begin{array}{cc}
\sigma^{2} & \text { if } n=1, \\
\sigma^{2}\left[1+\sum_{j=1}^{n-1}\left(\mathbf{w}^{\mathrm{T}} \mathbf{F}^{j-1} \mathbf{g}\right)^{2}\right] & \text { if } n \geq 2 .
\end{array}\right.
\end{aligned}
$$

As demonstrated in [38], point forecasts and forecast intervals are obtained using the inverse Box-Cox transformation.

Taking the $n_{d}$ time series of demands in the Barcelona DWN, and computing the inverse transformation of (C.9) and (C.10) applied to each of them, it is possible to define the elements that complete the stochastic properties of the DWN model as follows:

$$
\begin{aligned}
\overline{\mathbf{d}}_{k+n \mid k} & \triangleq\left[\bar{d}_{1, k+n \mid k}, \ldots, \bar{d}_{n_{d}, k+n \mid k}\right]^{\mathrm{T}}, \\
\Sigma_{\mathbf{d}, k+n \mid k} & \triangleq \operatorname{diag}\left(\Sigma_{d(1), k+n \mid k}, \ldots, \Sigma_{d\left(n_{d}\right), k+n \mid k}\right), \quad \forall n \in \mathbb{N}_{1}^{H_{p}} .
\end{aligned}
$$

\section{Acknowledgements}

This work has been partially supported by the EU Project EFFINET (FP7-ICT-2011-8-31855) and the DGR of Generalitat de Catalunya (SAC group Ref. 2009/SGR/1491).

\section{References}

[1] H. Alegre, J.M. Baptista, E. Cabrera Jr., and F. Cubillo. Performance Indicators for Water Supply Services. Manuals of Best Practice Series. IWA Publishing, 2006. 
[2] A. Castelletti, F. Pianosi, and R. Soncini-Sessa. Stochastic and robust control of water resource systems: Concepts, methods and applications. In System Identification, Environmental Modelling, and Control System Design, pages 383-401. Springer London, 2012.

[3] I. Pulido-Calvo and J.C. Gutiérrez-Estrada. Selection and operation of pumping stations of water distribution systems. Envirnomental Research Journal, 5:1-20, 2011.

[4] V. Nitivattananon, E. Sadowski, and R. Quimpo. Optimization of water supply system operation. Journal of Water Resources Planning and Management, 122(5):374-384, 1996.

[5] A. Shepherd and L. Ortolano. Water-supply system operations: Critiquing expert-system approach. Journal of Water Resources Planning and Management, 122(5):348-355, 1996.

[6] P. Crawley and G. Dandy. Optimal operation of multiple reservoir system. Journal of Water Resources Planning and Management, 119(1):1-17, 1993.

[7] A. El Mouatasim, R. Ellaia, and A. Al-Hossain. A continuous approach to combinatorial optimization: application of water system pump operations. Optimization Letters, 6:177-198, 2012. 
[8] C. Biscos, M. Mulholland, M-V Le Lann, C.A. Buckley, and C.J. Brouckaert. Optimal operation of water distribution networks by predictive control using MINLP. Water SA, 29:393-404, 2003.

[9] G. Cembrano, G. Wells, J. Quevedo, R. Pérez, and R. Argelaguet. Optimal control of a water distribution network in a supervisory control system. Control Engineering Practice, 8(10):1177-1188, 2000.

[10] J. Pascual, J. Romera, V. Puig, R. Creus, and M. Minoves. Operational predictive optimal control of Barcelona water transport network. In Proc. 18th IFAC World Congress, pages 10571-10578, Milano, Italy, 2011.

[11] V. Puig, C. Ocampo-Martínez, and S. Montes. Hierarchical temporal multi-layer decentralised MPC strategy for drinking water networks: application to the barcelona case study. In 2012 Mediterranean Conference on Control and Automation (MED), pages 740-745, Barcelona, Spain, 2012.

[12] J.M. Grosso, C. Ocampo-Martínez, and V. Puig. Learning-based tuning of supervisory model predictive control for drinking water networks. Engineering Applications of Artificial Intelligence, 26(7):1741-1750, 2013. 
[13] J.M. Maciejowski. Predictive control with constraints. Prentice Hall, Essex, England, 2002.

[14] C. Ocampo-Martínez, V. Puig, G. Cembrano, and J. Quevedo. Application of predictive control strategies to the management of complex networks in the urban water cycle. Control Systems, IEEE, 33(1):15-41, 2013.

[15] L. Chisci, P. Falugi, and G. Zappa. Predictive control for constrained systems with polytopic uncertainty. In Proc. of the 2001 American Control Conference (ACC), volume 4, pages 3073-3078, Arlington, Virginia, USA, 2001.

[16] Paul J. Goulart, Eric C. Kerrigan, and Jan M. Maciejowski. Optimization over state feedback policies for robust control with constraints. Automatica, 42(4):523-533, 2006.

[17] J.H. Lee and Zhenghong Yu. Worst-case formulations of model predictive control for systems with bounded parameters. Automatica, 33(5):763-781, 1997.

[18] D.Q. Mayne, J.B. Rawlings, C.V. Rao, and P.O.M. Scokaert. Constrained model predictive control: Stability and optimality. Automatica, 36(6):789-814, 2000. 
[19] S.V. Rakovic, B. Kouvaritakis, M. Cannon, C. Panos, and R. Findeisen. Parameterized tube model predictive control. IEEE Transactions on Automatic Control, 57(11):2746-2761, 2012.

[20] G. Calafiore and F. Dabbene. Probabilistic and randomized methods for design under uncertainty. Springer, 2006.

[21] A.T. Schwarm and M. Nikolaou. Chance-constrained model predictive control. AIChE Journal, 45(8):1743-1752, 1999.

[22] A. Charnes and W. W. Cooper. Chance-constrained programming. Management Science, 6:73-79, 1959.

[23] A. Geletu, M. Klöppel, H. Zhang, and P. Li. Advances and applications of chance-constrained approaches to systems optimisation under uncertainty. International Journal of Systems Science, 44(7):1209-1232, 2013.

[24] T.B.M.J. Ouarda and J.W. Labadie. Chance-constrained optimal control for multireservoir system optimization and risk analysis. Stochastic Environmental Research and Risk Assessment, 15:185-204, 2001.

[25] J. Matusko and F. Borrelli. Scenario-based approach to stochastic linear 
predictive control. In Proc. 51st IEEE Annual Conference on Decision and Control (CDC), pages 5194-5199, Maui, Hawaii, USA, 2012.

[26] D. Muñoz de la Peña, A. Bemporad, and T. Alamo. Stochastic programming applied to model predictive control. In Proc. 44th IEEE Conference on Decision and Control and 2005 European Control Conference (CDC-ECC), pages 1361-1366, Seville, Spain, 2005.

[27] J.M. Grosso, C. Ocampo-Martínez, and V. Puig. A service reliability model predictive control with dynamic safety stocks and actuators health monitoring for drinking water networks. In 51st IEEE Annual Conference on Decision and Control (CDC), pages 4568-4573, Maui, Hawaii, USA, 2012.

[28] C. Ocampo-Martínez, V. Puig, J.M. Grosso, and S. Montes-deOca. Multi-layer decentralized MPC of large-scale networked systems. In Distributed Model Predictive Control Made Easy, pages 495-515. Springer, 2014.

[29] B. Joseph and C. Ocampo-Martínez. Reduction of variables in model predictive control problems with equality constraints. Technical Report IRI-TR-09-07, Institut de Robòtica i Informàtica Industrial, CSIC-UPC, 2009. (In Catalan). 
[30] M.P. Vitus and C.J. Tomlin. On feedback design and risk allocation in chance constrained control. In Proc. 50th IEEE Conference on Decision and Control and European Control Conference (CDC-ECC), pages 734739, Orlando, Florida, USA, 2011.

[31] P. Kall and J. Mayer. Stochastic linear programming. Springer, 2005.

[32] E. Cinquemani, M. Agarwal, D. Chatterjee, and J. Lygeros. Convexity and convex approximations of discrete-time stochastic control problems with constraints. Automatica, 47(9):2082-2087, 2011.

[33] A. Prekopa. Stochastic Programming. Kluwer Academic Publishers, 1995.

[34] A. Nemirovski and A. Shapiro. Convex approximations of chance constrained programs. SIAM Journal on Optimization, 17(4):969-996, 2006.

[35] M. Ono and B.C. Williams. Iterative risk allocation: A new approach to robust model predictive control with a joint chance constraint. In Proc. 47th IEEE Conference on Decision and Control, pages 3427-3432, Cancun, Mexico, 2008.

[36] A. Charnes and W. W. Cooper. Deterministic equivalents for optimizing 
and satisficing under chance constraints. Operations Research, 11(1):1839, 1963.

[37] C. Ocampo-Martínez, V. Puig, G. Cembrano, R. Creus, and M. Minoves. Improving water management efficiency by using optimization-based control strategies: the Barcelona case study. Water Science $\&$ Technology: Water supply, 9(5):565-575, 2009.

[38] A.M. De Livera, R.J. Hyndman, and R.D. Snyder. Forecasting time series with complex seasonal patterns using exponential smoothing. Journal of the American Statistical Association, 106(496):1513-1527, 2011. 